\title{
FOCALIZADORES ADITIVOS ESCALARES Y POSICIÓN ENUNCIATIVA: UN ESTUDIO CONTRASTIVO ESPAÑOL / ITALIANO ${ }^{1}$
}

\author{
Margarita Borreguero Zuloaga \\ Universidad Complutense de Madrid
}

\begin{abstract}
Resumen: La correspondencia entre los focalizadores aditivos escalares de polaridad positiva del español (incluso, aun, hasta) y del italiano (perfino/persino, addirittura, anche) es un ámbito del estudio contrastivo entre ambas lenguas que merece mayor atención. En este trabajo se propone un análisis de las funciones discursivas de incluso con la ayuda de un modelo de unidades discursivas (el Modelo de Basilea) con el objetivo de identificar cuáles son sus equivalentes italianos más adecuados en cada caso. Se trata de un estudio basado en dos corpus de textos periodísticos del que se deduce que el equivalente italiano que se corresponde de manera más fiel con incluso es addirittura, mientras que perfino/persino tiene un uso más restringido a los casos en que incluso focaliza un elemento presente en el enunciado.
\end{abstract}

Palabras clave: focalizadores escalares, conectores aditivos, incluso, addirittura, perfino/persino, Modelo de Basilea

Abstract: The correspondence between the scalar additive focus adverbs in Spanish (incluso, aun, hasta) and Italian (perfino/persino, addirittura, anche) deserves further research in contrastive studies. In this paper we propose an analysis of the discursive functions of incluso 'even', which are described with the help of a model of discursive units (the Basel Model). Our aim is to identify which are the most adequate Italian equivalents in each case. After conducting a corpus-based study on journalistic texts we come to the conclusion that addirittura covers most of the functions of incluso, while perfino/persino is restricted to the focusing of an element which is present in the utterance.

Keywords: scalar focus particles, additive connectives, incluso, addirittura, perfino/persino, Basel Model

${ }^{1}$ Este trabajo se enmarca en el proyecto Estructura informativa y marcación discursiva en la didáctica de la oralidad en italiano y español L2 (FFI2011-24960), financiado por el Ministerio de Economía y Competitividad, del que la autora es investigador principal. 
Los focalizadores han sido un objeto clásico de estudio en la teoría lingüística desde que despertaron el interés de los estudiosos de la semántica formal por su posibilidad de modificar las condiciones de verdad de un enunciado según la posición que ocuparan dentro de este (cf. König 1993 para una introducción general). A partir de entonces, y en paralelo al desarrollo de la teoría general del foco, se han sucedido los estudios que han tratado de acercarse a estas unidades desde perspectivas sintácticas, semánticas y pragmáticas y desde enfoques generativos, funcionales y cognitivos. Más tarde se desarrolló una línea de investigación que analizaba los focalizadores desde la perspectiva del contraste lingüístico, línea cuya obra más representativa es The meaning of focus particles: a comparative perspective (1991) de Ekkehard König, pero véase también Gast / van der Auwera (2011). Este trabajo se sitúa en este campo de investigación y continúa el camino iniciado por otros estudios sobre los focalizadores aditivos en las lenguas románicas desde un enfoque comparado (Lenarduzzi 1995, García Negroni 1998, Sainz 2006a/b, Borreguero 2011, De Cesare/Borreguero 2014).

En esta ocasión nuestro interés se centra en el focalizador aditivo escalar incluso y en sus equivalentes en la lengua italiana. Desde la perspectiva textual en la que se sitúa nuestra investigación, nos interesa especialmente descubrir los distintos valores semántico-pragmáticos del focalizador que emergen a partir de su inserción en el texto, es decir, de la posición que ocupa en la estructura del enunciado -que condiciona su alcance y, por tanto, su radio de acción sobre otros elementos enunciativos- y del modo como dicha posición interactúa con el valor semántico nuclear del focalizador y con las funciones textuales que adquiere en los distintos contextos. Pretendemos de este modo mostrar, en primer lugar, la riqueza semántica y funcional de estas unidades que va más allá de la función focalizadora que le atribuyen los enfoques sintácticos y semánticos y, en segundo lugar, la consiguiente complejidad a la hora de encontrar equivalentes en una lengua afín como el italiano y las limitaciones (inevitables) de las soluciones lexicográficas que, en este caso, se restringen por lo general a persino/perfino, addirittura y anche sin mayores profundizaciones (cf. Tam 1997, Arquès / Padoan 20122).

Para llevar a cabo dicho estudio nos ha resultado especialmente útil partir de un modelo de unidades discursivas, es decir, de un modelo de análisis textual que, considerando el enunciado como la unidad textual básica, identifique en su estructura segmentos que responden a determinadas propiedades topográficas, lingüísticas e informativas y que establecen entre sí relaciones jerárquicas. Dado que nuestra investigación parte del análisis de textos periodísticos,

${ }^{2}$ Este último diccionario establece una diferencia categorial entre incluso como adjetivo (equivalente: compreso, incluso), como adverbio (equivalente perfino, persino, addirittura, anche), como preposición (equivalente: anche, persino) y conjunción (equivalente: anche), si bien la diferencia entre las tres últimas categorías no es, a nuestro juicio, suficientemente clara. Cf. Arquès / Padoan 2012: s.v. incluso. 
nos ha parecido apropiado adoptar como marco teórico el único modelo de unidades discursivas que, hasta donde sabemos, se ha preocupado de describir la estructura informativa del texto escrito: el llamado modelo de $\mathrm{Basilea}^{3}$, que presentaremos brevemente en el siguiente epígrafe, y cuya rentabilidad para el análisis lingüístico esperamos poder corroborar también a través de este estudio.

\section{LA INTERACCIÓN ENTRE LOS VALORES SEMÁNTICO-PRAGMÁTICOS Y LA POSICIÓN ENUNCIATIVA: UNA BREVE PRESENTACIÓNDEL MODELO DE BASILEA}

Los modelos de unidades discursivas que se han desarrollado a partir de la década de los noventa, en un intento por superar algunas limitaciones analíticas de la teoría sintáctica a la hora de explicar la estructura de los enunciados en la interacción, pero también de la teoría clásica praguense que describió por primera vez la estructura informativa del enunciado, han permitido identificar los valores semántico-pragmáticos de los elementos lingǘsticos en su funcionamiento dentro un determinado texto, adoptando una perspectiva que Angela Ferrari y sus colaboradores denominaron con término afortunado "la interfaz" entre la lengua y el texto (cf. Ferrari et al. 2008).

Como hemos adelantado, de los distintos modelos de unidades discursivas, elaborados en su mayoría en el ámbito francófono ${ }^{4}$, servirá de marco teórico a este estudio el llamado modelo de Basilea (Ferrari 2003, Ferrari (ed.) 2004, 2005, Ferrari et al. 2008, entre otros; cf. la traducción y adaptación al español en Ferrari/Borreguero 2015). El modelo de Basilea, que se diferencia de los citados (véanse notas 3 y 4 ) porque se presenta como un modelo diseñado para el análisis del texto escrito, contempla tres niveles de organización del texto a los que denomina respectivamente:

1. Nivel tópico: cómo se introducen en el texto y se desarrollan a lo largo de este los sucesivos tópicos discursivos que constituyen el universo del discurso de cada texto.

${ }^{3}$ El modelo ha sido elaborado por Angela Ferrari y sus diversos colaboradores durante el desempeño de su actividad docente e investigadora en las universidades de Losana y Basilea. Dado que ha sido durante su permanencia en esta última universidad cuando se ha publicado la mayor parte de los libros y artículos en los que se describe y se aplica el modelo textual, y especialmente la obra que hoy por hoy constituye su descripción más completa, La interfaccia lingua-testo. Natura e funzioni dell'articolazione informativa dell'enuciato (2008), pronto se adoptó el nombre del Modelo de Basilea, quizá por paralelismo con otras denominaciones en este campo como el modelo o teoría modular de la Escuela de Ginebra, el modelo del GARS (Gruppe Aixoise de Recherche Scientifique), etc.

${ }^{4}$ Entre los más conocidos, destacamos el de Roulet y sus colaboradores (1985, 1991), que ha sido después adaptado para el español por el grupo Val.Es.Co. (Briz y Val.Es.Co. 2003, entre otros) y por Cortés y Camacho (2002), el de Degand y sus colaboradores y el de Morel, que tiene en cuenta sobre todo elementos prosódicos y mímicos. Para una presentación de conjunto de muchos estos modelos, cf. Pons (2014). 
2. Nivel lógico-argumentativo: cómo se presentan distintos argumentos y contraargumentos para guiar al receptor hacia una conclusión determinada y cuáles son las relaciones lógicas que se establecen entre ellos.

3. Nivel jerárquico-ilocutivo: cómo se estructura la información presentada en el texto, es decir, cómo se pone de relieve la información principal y cómo se subordina a esta información secundaria o marginal.

En este trabajo nos interesa fundamentalmente este último nivel y no nos ocuparemos, por lo general, de los otros dos. El punto de partida de la propuesta de estructura jerárquico-informativa en el texto lo constituye el análisis del enunciado que llevó a cabo Claire Blanche-Benveniste $(1990,1994)$ en sus estudios sobre el francés oral, aunque integra también los fundamentos de la teoría de actos de habla y de la lingüística textual. La estudiosa francesa dividió el enunciado en tres unidades, determinadas topográficamente por su ubicación dentro de este, con funciones estructural e informativamente distintas: de izquierda a derecha, preffix, noyau, suffix $x^{5}$.

Ferrari distingue dos niveles estructurales en el texto:

a) Las Unidades Comunicativas o unidades básicas semántico-pragmáticas de la estructura del texto, en las que a su vez puede distinguirse un significado implícito y un significado explícito. Este último se manifiesta verbalmente en un texto bajo la forma de una proposición, un conjunto de proposiciones o una estructura infraproposicional delimitados por signos de puntuación fuerte y recibe el nombre de Enunciado $^{6}$.

(1) // Un hombre de 31 años murió víctima de una brutal paliza propinada por un grupo de agresores en la madrugada del pasado sábado a la salida de un local nocturno de Barcelona.// $\mathrm{E} 1 \mathrm{El}$ herido falleció horas después de los hechos a consecuencia de las lesiones internas causadas por los numerosos golpes y patadas recibidos, tras haber sido dado de alta en el hospital Clínico de Barcelona, según explicaron ayer fuentes policiales. // Е2 (Diario Vasco, $14 / 12 / 2000)^{7}$

${ }^{5} \mathrm{El}$ modelo de Blanche-Benveniste ha sido adaptado y enriquecido para el estudio del italiano oral por Cresti (2000) y sus colaboradores en el Laboratorio de Lengua Italiana (Lablita) de la Universidad de Florencia. En los últimos años, este modelo se ha aplicado a otras lenguas como el portugués.

${ }^{6} \mathrm{El}$ uso de las mayúsculas indica que se trata de los términos propios del modelo (en su traducción española) con un sentido preciso y bien determinado que no siempre coincide con el que estos términos tienen en otros marcos teóricos. Las fronteras entre Enunciados aparecen marcadas por barras dobles (//) y los Enunciados van numerados consecutivamente. Este número aparece en subíndice a la derecha de la doble barra: // 1 .

${ }^{7}$ Los ejemplos que presentaremos están extraídos fundamentalmente de dos corpus: a) para el español, el Corpus CREA (Corpus de Referencia del Español Actual) de la RAE, subcorpus de textos periodísticos publicados en España en el periodo comprendido entre los años 19942004 (últimos años para los que se recogieron textos en este subcorpus), aunque en algunos casos excepcionales hemos recurrido a textos publicados con anterioridad a este periodo; b) para el italiano, el Corpus CORIS (Corpus di Italiano Scritto), de la Universidad de Bolonia, 
b) Las Unidades Informativas son las unidades en que se puede dividir un Enunciado y en la última versión del modelo son únicamente tres: el Marco, el Núcleo y el Apéndice (nótese, por tanto, el paralelismo con la propuesta de Blanche-Benveniste, aunque como veremos hay diferencias importantes) ${ }^{8}$. En el texto escrito, normalmente las fronteras entre Unidades Informativas están marcadas por signos de puntuación, aunque también se tienen en cuenta otros criterios relativos a la estructura sintáctica y al contenido semántico. La única Unidad obligatoria y necesaria para poder hablar de Enunciado es el Núcleo (el noyau de Blanche-Benveniste), Unidad que vehicula el contenido semántico fundamental del enunciado y que indica su modalidad ilocutiva, es decir, el tipo de acto lingüístico que se lleva a cabo por medio del enunciado.

(2) // Un hombre de 31 años murió víctima de una brutal paliza propinada por un grupo de agresores en la madrugada del pasado sábado a la salida de un local nocturno de Barcelona./Núcleo // $E 1$ El herido falleció horas después de los hechos a consecuencia de las lesiones internas causadas por los numerosos golpes y patadas recibidos, $/$ Núcleo tras haber sido dado de alta en el hospital Clínico de Barcelona, según explicaron ayer fuentes policiales. // E2

Opcionalmente, dicho Núcleo puede ir precedido de un Marco (preffix en Blanche-Benveniste, Quadro en Ferrari), una Unidad que normalmente contiene información relativa al ámbito en el que es pertinente la información del Núcleo (circunstancias temporales, espaciales y modales, introducción o recuperación de un tópico por medio de un tematizador) o a la fuente de que se extrae, o bien un elemento que sirve de unión con el Enunciado precedente y marca el tipo de relación metadiscursiva o lógico-argumentativa que se establece entre ambos Enunciados 9 .

(3) Según testigos presenciales,/Marco Abu Saui, de 28 años, fue tiroteado sin que en las cercanías tuviera lugar algún enfrentamiento con soldados israelíes, lo que apoya la versión de que se trató de un asesinato premeditado y selectivo de los servicios secretos de Israel, que ya han efectuado actos similares contra líderes de la intifada en las últimas semanas. // $\mathrm{E} 1$

Por otro lado, /Marco1 en diversos puntos de Cisjordania y Gaza/Marco2 se registraron ayer otros incidentes entre palestinos y soldados israelíes, aunque sin víctimas mortales. // E2 (La Voz de Galicia, 13/12/2000)

con exclusión de los textos literarios (en este caso no es posible precisar la fecha, ya que no se especifica en las búsquedas del corpus; en la introducción se indica de manera genérica que los textos pertenecen a la década de los 80 y los 90 , aunque ha sido completado posteriormente con textos del año 2001,2004 y 2008). Mientras en el caso del CREA es posible indicar con exactitud la fuente de la que está extraído cada ejemplo, en el caso del CORIS tenemos que conformarnos con indicar el subcorpus al que pertenece. Es posible consultar ambos corpus en libre acceso en Internet.

${ }^{8}$ Las Unidades Informativa se separan en el análisis textual por una barra simple (/) y el tipo al que pertenecen se indica en superíndice a la derecha de la barra: /Núcleo.

${ }^{9}$ Sobre la Unidad de Marco, que ha sido la que más vaivenes conceptuales y terminológicos ha sufrido durante la elaboración del modelo, véase Zampese (2005) y Borreguero (2014). 
Por último, la Unidad de Apéndice (también opcional) es la que presenta mayor movilidad, ya que puede aparecer bien a continuación del Núcleo o del Marco o bien inserta en cualquiera de estas dos Unidades Informativas. Su contenido semántico normalmente aclara, precisa o puntualiza alguna de las informaciones aparecidas en otras Unidades del Enunciado, aunque también puede contener elementos funcionales como los conectores, como veremos. La principal diferencia con la Unidad de Marco es que, debido a su posición, aunque el contenido semántico puede ser muy similar, en el caso del Apéndice su validez se restringe al Núcleo junto al que aparece, mientras que en el caso del Marco, la posición inicial le permite mantener en vigor la información no solo para el Núcleo que precede sino para todo el Enunciado e incluso para un conjunto de Enunciados. En (4) presentamos un ejemplo de un Apéndice inserto en la Unidad de Núcleo y dos ejemplos de Apéndices que siguen a la Unidad de Núcleo (Apéndices extensivos) y que se subordinan informativamente el uno al otro (por tanto, podemos hablar también de un Apéndice de un Apéndice). Como se ve, estos últimos, por su posición final, suelen ser más amplios.

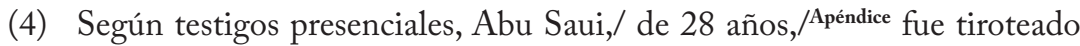
sin que en las cercanías tuviera lugar algún enfrentamiento con soldados israelíes,/ lo que apoya la versión de que se trató de un asesinato premeditado y selectivo de los servicios secretos de Israel,/Apéndice $q u e$ ya han efectuado actos similares contra líderes de la intifada en las últimas semanas./Apéndice // $\mathrm{E} 1$

Si analizamos todas las Unidades Informativas de los Enunciados de los dos breves fragmentos textuales citados, el resultado sería el siguiente:

(5) // Un hombre de 31 años murió víctima de una brutal paliza propinada por un grupo de agresores en la madrugada del pasado sábado a la salida de un local nocturno de Barcelona./Núcleo // $\mathrm{E} 1 \mathrm{El}$ herido falleció horas después de los hechos a consecuencia de las lesiones internas causadas por los numerosos golpes y patadas recibidos, ${ }^{\text {Núcleo }}$ tras haber sido dado de alta en el hospital Clínico de Barcelona,/Apéndice según explicaron ayer fuentes policiales./Apéndice // ${ }_{\mathrm{E} 2}$

(6) // Según testigos presenciales,/Marco Abu Saui,/Núcleo- de 28 años,/Apéndice fue tiroteado sin que en las cercanías tuviera lugar algún enfrentamiento con soldados israelíes, $/$-Núcleo lo que apoya la versión de que se trató de un asesinato premeditado y selectivo de los servicios secretos de Israel,/Apéndice que ya han efectuado actos similares contra líderes de la intifada en las últimas semanas./Apéndice // $\mathrm{E} 1$

Por otro lado, $/{ }^{\text {Marco1 }}$ en diversos puntos de Cisjordania y Gaza/Marco2 se registraron ayer otros incidentes entre palestinos y soldados israelíes,/Núcleo aunque sin víctimas mortales./Apéndice // E2

A partir de este marco teórico, delineado aquí muy someramente, nos proponemos analizar los valores semántico-pragmáticos que un mismo 
elemento puede adquirir según la Unidad Informativa en que se encuentre y según el material lingüístico que lo acompañe, es decir, si dicho elemento aparece saturando por sí solo la Unidad Informativa o modificando o siendo modificado por otros.

\section{PROPIEDADES SEMÁNTICAS Y SINTÁCTICAS DE LOS FOCALIZADORES} ADITIVOS ESCALARES

La elección de un focalizador como objeto de estudio no solo se debe a nuestros propios intereses de investigación que, como hemos adelantado, han versado sobre el análisis de estos elementos desde una perspectiva contrastiva (Borreguero 2011, 2012, De Cesare/Borreguero 2014), sino también al hecho de que estos elementos, junto con los conectores y otros marcadores del discurso, han recibido particular atención entre los autores del modelo de Basilea y ha sido uno de los campos en los que se ha puesto de relieve la rentabilidad analítica de dicho modelo. Así, por ejemplo, han sido muy numerosos los estudios que Anna-Maria De Cesare ha dedicado a los focalizadores tanto identificativos - proprio 'precisamente', davvero y veramente 'realmente, verdaderamente' (De Cesare 2002a/b, Ferrari / De Cesare 2004) - como particularizadores - soprattutto 'sobre todo' (De Cesare 2006, 2008a) y aditivos (cf. De Cesare 2004a/b, 2009, sobre anche $)^{10}$-.

La atención que se ha prestado a los focalizadores aditivos escalares ha sido desigual en ambas lenguas. En español contamos con un buen número de estudios sobre incluso, que van desde acercamientos a sus propiedades sintácticas y semánticas desde un enfoque estructural tradicional (Cuartero Sánchez 2002) hasta aproximaciones semánticas en el marco de la Teoría de la Argumentación (Fuentes 1987, García Negroni 1998, Garrido 1993)ํ․ Para lo que aquí nos interesa, resultan, sin embargo, más interesantes los estudios de corte pragmático (Schwenter 2000, Portolés 2009, 2010), siguiendo la teoría de la semántica de las alternativas de Rooth (1985), de la que hablaremos más

${ }^{10}$ En cuanto a los conectores, además de algunas visiones de conjunto (Ferrari / Mandelli 2007, Ferrari 2008, Ricci 2008), destacan estudios como Mandelli $(2004,2006)$ sobre los valores discursivos de $e$ 'y', Ferrari (2005) para dunque 'por tanto', Ferrari $(2008,2015)$ para dunque / quindi; también en perspectiva contrastiva con el francés (Ferrari / Rossari 1994 para donc/dunquel quindi, Ferrari / Ricci 2011) o con el español (cf. Borreguero / López Serena 2011 sobre allora / entonces, Guil 2014 sobre quindi/por tanto). De los elementos con función interaccional han recibido especial atención aquellos con valores de confirmación (cf. Ricci 2008 sobre effettivamente y Mandelli 2008 sobre in effetti 'efectivamente').

${ }^{11}$ No tendremos en cuenta en este estudio otros focalizadores aditivos escalares del español, como hasta y aun, aunque sí podemos señalar que la principal diferencia semántica con incluso en el caso de hasta reside en que indica el elemento culminativo de la escala, de modo que no puede añadirse ningún otro argumento que, perteneciendo a la misma escala, ocupe un lugar por encima de él (para un estudio más en profundidad sobre ambos focalizadores, véase Fuentes 1987). Y en cuanto a aun, además de las diferencias de registro, tiene una movilidad más reducida en el enunciado y no puede funcionar en ningún contexto como conector aditivo. 
abajo, que se han completado recientemente con nuevos enfoques cognitivoexperimentales (Loureda et al. 2012, Loureda 2014). Para el italiano conocemos, además de algunas observaciones en estudios de alcance más general (Ricca 1999, Andorno 2000, La Forgia 2006), varios trabajos de De Cesare sobre anche (De Cesare 2004a/b, 2009, 2010), un estudio sobre persino/perfino en el marco de la teoría de la argumentación (Schiemann 2009) y dos trabajos de Visconti $(2004,2005)$ sobre persino/perfino y addirittutra, que tratan sobre todo acerca de su origen histórico ${ }^{12}$. Tampoco se encuentran estudios contrastivos sobre los adverbios focalizadores italianos y españoles (excepto los mencionados en el párrafo anterior), a pesar de que existen diversos trabajos dedicados al contraste de otros tipos de adverbios: temporales (Lenarduzzi 1997a/b), dubitativos (Bermejo 2007) y al adverbio en general (Bermejo 2013). Por ello, hemos creído más conveniente partir del adverbio español y de sus descripciones semánticas $\mathrm{y}$ funcionales para establecer en cada caso las correspondencias con los posibles equivalentes en italiano ${ }^{13}$.

En el Diccionario de la Real Academia Española se adscribe incluso a tres categorías gramaticales: la de adjetivo (que no tendremos en cuenta aquí), la de adverbio modal y la de preposición. Sin entrar en estas diferencias categoriales ${ }^{14}$,

${ }^{12}$ La bibliografía es, por el contrario, muy extensa para el inglés even (cf., entre otros, Kay 1990, Wilkinson 1996, Traugott 2006) y para el francés même (cf. Anscombre / Ducrot [1983]1994, Nølke 1983 por citar solo las referencias más emblemáticas). Véase la bibliografía citada en Fuentes (1987).

${ }^{13}$ Incluso no es el único focalizador aditivo escalar de polaridad positiva en español, aunque sí el más frecuente. Tienen valor escalar indiscutible el adverbio aun (y el menos usado todavía) y la preposición hasta, aunque discursivamente su comportamiento difiere del de incluso (Fuentes 1997). También son escalares los adverbios además, encima, y la locución adverbial a más de esto, que funcionan habitualmente como conectores parentéticos y, por tanto, son equivalentes de incluso solo en determinados contextos. En este trabajo nos apoyaremos en el análisis de incluso que hemos realizado en Ferrari/Borreguero (2015).

${ }^{14}$ Hoy pocos estudiosos estarían de acuerdo con el hecho de que incluso actúa como preposición en los casos en que desempeña la función discursiva de focalizador, que veremos más adelante (véase al respecto Cuartero Sánchez 2002, Santos Río 2003: 418). Sin embargo, es en estos usos (sinónimos de hasta y aun) en los que el DRAE considera incluso preposición y no adverbio (en efecto, el ejemplo es Incluso a los enemigos amó). La distinción es especialmente compleja ya que el DRAE no ofrece ningún ejemplo del uso adverbial, limitándose a definirlo por medio de los sinónimos con inclusión, inclusivamente. En cualquier caso, en la NGLE se dice ya explícitamente que «Si bien las partículas excepto, menos y salvo (§ 31.12) se han considerado a veces preposiciones, no suelen clasificarse actualmente como tales, y se asimilan más bien a las conjunciones [...]. Se clasifican como adverbios o locuciones adverbiales incluso (§ 40.8), sin embargo y no obstante (§ 30.12)» (NGLE 2009 § 29.2ñ). Aunque en distintas gramáticas incluso se ha considerado preposición, conjunción y hasta adverbio de modo (v. Cuartero Sánchez 2002: 62-81, 92-96 respectivamente para una revisión de las principales gramáticas y diccionarios de la $2^{\text {a }}$ mitad del s. XX y para una crítica a la adscripción de incluso a estas categorías en el español contemporáneo), hoy se acepta generalmente que pertenece a la categoría gramatical de los adverbios y dentro de esta a los llamados adverbios de foco (NGLE 2009 § 40.8). 
aquí analizaremos fundamentalmente las dos funciones discursivas más habituales de incluso como adverbio focalizador y como conector.

$\mathrm{Si}$ atendemos en primer lugar a sus propiedades semánticas, lo primero que hay que señalar es que el valor nuclear ${ }^{15}$ de incluso $-y$ con nuclear queremos indicar que permanece a pesar de los cambios funcionales que experimenta en los distintos contextos- es aditivo, ya que indica que un elemento se añade a una serie de elementos similares, que pueden aparecer de forma explícita en el texto o permanecer implícitos si se considera que son fácilmente recuperables por el destinatario.

En tanto que adverbio focalizador, su función principal es la de indicar que el elemento al que precede (o, más raramente, al que se pospone) constituye el segmento informativamente más relevante en un determinado enunciado ${ }^{16}$. Esta función de focalización es compartida por un conjunto amplio pero finito de elementos en la lengua española, entre los que se cuentan también, hasta, solo, sobre todo, especialmente, etc. En cuanto a sus propiedades sintácticas, los adverbios focalizadores se consideran elementos transcategoriales (cross-categorial), ya que el elemento que focalizan puede pertenecer a cualquier tipo de sintagma (nominal, verbal, adjetival, adverbial o preposicional), como ponen de manifiesto los siguientes ejemplos: Hemos comprado incluso pasteles (SN), Incluso cantaron habaneras (SV), Fueron poco inteligentes, incluso estúpidos, al rechazar tal oferta (SAdj), Durmieron tranquilamente, incluso placenteramente (SAdv), Visitó incluso a los parientes más lejanos (SPrep). Asimismo, no existen tampoco restricciones respecto de la función sintáctica que puede desempeñar el elemento focalizado (Cuartero Sánchez 2002; Ferrari et al. 2011). Sin embargo, es difícil encontrar un uso en el que incluso pueda ser considerado adverbio de modo, tal y como aparece descrito en el DRAE.

Un adverbio focalizador crea un contraste entre el elemento que focaliza y una serie de elementos similares (por su significado, categoría, etc.) que constituyen una alternativa (Rooth 1985), en la medida en que establecen con el elemento

15 Seguimos la distinción establecida por Blakemore (1989) entre significado conceptual y significado procedimental y la postulada por Aijmer / Simon-Vandenbergen (2011) entre significado nuclear y significado pragmático. La peculiaridad de estos elementos que funcionan sobre todo en el plano discursivo (ya se les llame marcadores del discurso o partículas discursivas, con un término más amplio que incluye también a los focalizadores, cf. Briz et al. 2008) es que su significado nuclear tiene carácter procedimental o instruccional, mientras su significado pragmático puede variar según el tipo de función textual. Así, típicamente, cuando pertenece a una categoría gramatical bien definida (verbo, adjetivo, adverbio, etc.), una unidad léxica posee un significado nuclear de carácter conceptual, que se pierde total o parcialmente cuando dicha unidad asume la función de marcador discursivo, en un proceso de desemantización que es característico del proceso de gramaticalización que explica la emergencia de los marcadores a partir de determinadas categorías gramaticales (Hopper / Traugott 1993).

${ }^{16}$ Para una concepción diversa sobre la función focalizadora de estos adverbios, ligada al foco informativo del enunciado, véase De Cesare (2010). 
focalizado una relación paradigmática ${ }^{17}$. Los focalizadores se han clasificado tradicionalmente en dos grandes grupos: los exclusivos, que indican que el elemento focalizado es el único afectado por la información que se contiene en el predicado (ej. Solo Juan fue sincero implica que las demás personas que participaron en la misma situación comunicativa a la que hace referencia el enunciado no dijeron la verdad); y los aditivos, donde la información del enunciado mantiene su validez tanto para el elemento focalizado como para los que conforman la alternativa (ej. Luisa, Marta y también Esther son estudiantes brillantes) ${ }^{18}$.

Incluso pertenece a este segundo grupo de adverbios focalizadores aditivos, pero se diferencia de también en que no solo establece una relación de adición entre el constituyente focalizado y los que integran la alternativa, sino que además imprime un valor escalar al paradigma que forman dichos elementos. En otras palabras, el segmento discursivo que cae bajo el alcance del focalizador incluso se sitúa en un lugar superior de una escala ordenada. El criterio para la ordenación de la escala es de tipo pragmático ${ }^{19}$, lo que quiere decir que las escalas solo tienen validez en una situación comunicativa concreta y se construyen a partir del conocimiento del mundo compartido por los participantes en dicha situación comunicativa. El constituyente focalizado representa siempre un participante, circunstancia o hecho poco esperable en el estado de cosas representado (Portolés 2007, 2008, 2009; Santos Río 2003: 418)²0.

Téngase en cuenta que esta diferencia semántica entre un valor aditivo y un valor aditivo escalar no siempre se corresponde con una diferencia en la unidad léxica que los vehicula. En este sentido, es paradigmático el caso del italiano,

17 Las relaciones paradigmáticas entre los elementos que conforman la alternativa y el elemento focalizado han llevado a algunos autores a denominar a estas unidades "adverbios paradigmatizantes" (Nølke 1983). Otros estudiosos han explicado esta relación en términos de presuposición: el uso de incluso presupone la existencia de otros elementos en el contexto susceptibles de entablar la misma relación sintáctica y semántica que el foco de este adverbio y dicha presuposición está ya lexicalizada, por lo que algunos autores consideran incluso un operador epistémico (Garrido Medina 1993).

18 Sin embargo, varios autores (Taglicht 1993, Ricca 1999: 146-148, Andorno 2000, Ferrari et al. 2011) consideran dos tipos adicionales de adverbios focalizadores: los particularizadores (particularmente, especialmente) y los identificadores (exactamente, precisamente), que otros estudiosos incluyen dentro de los aditivos en otros casos (König 1993: 979-980).

19 También es posible construir escalas basadas en criterios semánticos de gradación, como en el caso de Entre mis pacientes hay enfermos leves, moderados, avanzados e incluso en fase terminal. Incluso la gradación puede llegar a ser más transparente cuando se usa el mismo lexema para algunos grados de la escala: Mis alumnos se ponen a veces pesados, muy pesados, incluso pesadísimos a la hora de revisar sus exámenes.

20 Como hemos dicho, los focalizadores que tienen valor aditivo escalar en español son incluso, hasta y aun, mientras que este mismo valor se reconoce también en conectores con un alcance discursivo más amplio como es más y encima (para otros conectores y operadores discursivos que actúan también sobre un paradigma de alternativas, cf. Portolés 2009). Sin embargo, en determinados contextos también y además pueden adquirir valores escalares que no están codificados en la semántica de estos adverbios, sino que dependen estrechamente del contexto de aparición (cf. Borreguero 2011; Portolés 2013: 292). 
donde los adverbios anche y, en menor medida, pure pueden expresar tanto el valor aditivo simple (7-8) como el escalar (9-10)21:

(7) L'inizio di un nuovo anno scolastico è un avvenimento importante, per tutta la famiglia. Insieme al figlio, infatti, è un po' come se tornassero sui banchi anche mamma e papà. Che sono al suo fianco. $\mathrm{E}$ devono aiutarlo a risolvere piccoli e grandi problemi che spesso si presentano fin dai primi mesi di scuola. (CORIS, STAMPAPeridioci)

(8) Ora Capello, analizzando il primo posto in campionato, sei punti sopra il Barcellona, si rende conto dell'imprescindibilità di rinforzi pesanti, cinque almeno, per onorare al meglio la Champions League. Ma, e questa è l'autentica novità, ha colto nell'atteggiamento societario una decisa riluttanza ad ulteriori investimenti. Capello aspetta un segnale da Berlusconi ed è evidente che da Berlusconi dipenderebbe pure uneventuale intesa con l'Inter di Moratti. (CORIS, STAMPAQuotidiani)

(9) A meno di un mese dalla sua costruzione, abitanti e commercianti della zona lo bollano come il frutto di un pazzo e gli automobilisti costretti a scavalcarlo lo scrutano come si farebbe con un potenziale assassino. In effetti il dosso artificiale, costruito davanti alla piscina comunale e alla scuola L. Sinigaglia, anche di giorno ha un aspetto sinistro: oltre che esageratamente alto, il dissuasore è poco visibile e mal segnalato.(CORIS, MOND2001_04) (10) Sia nell'incontro con il presidente russo Medvedev per trattare della riduzione degli armamenti; sia nella presenza al G8, al quale indubbiamente ha dato un'impronta particolare e positiva; sia nella visita a Benedetto XVI, dove pure sono stati trattati temi irrinunciabili per la dottrina cattolica come la difesa della vita; sia, infine, nell'incontro in Ghana con gli africani attraverso un discorso veramente alto, da africano, Obama è sempre partito da una chiarezza assoluta delle posizioni. (CORIS, MOND2008_10)22

21 Tanto anche y pure como su equivalente también pueden desempeñar otras funciones discursivas distintas de la focalización, como, por ejemplo, la modalización del enunciado. Así, anche es un mecanismos de intensificación en Lo potevi anche dire prima che non venivi! y pure lo es de atenuación en Faccia pure con calma che io aspetto qua. Por su parte, también puede indicar la aceptación parcial de un enunciado (en un sentido próximo a la concesión) al hacer explícito un argumento que se contrapone a la inferencia esperada. Así en:

A: Es muy lista y muy estudiosa

B: También ha tenido siempre la vida resuelta y nunca ha tenido que trabajar

El hablante B argumenta que no todo el mérito depende de la persona a la que se refiere A y señala cómo le han favorecido las circunstancias (Sanz 2006b).

Pero, incluso en su función como focalizadores, hay importantes diferencias entre ellos de naturaleza semántica (el carácter más frecuentemente aditivo y escalar de anche, frente al simplemente aditivo de pure) y sintáctica (anche tiene mayor movilidad en el enunciado y se combina con una mayor variedad de constituyentes sintagmáticos, mientras que pure extiende habitualmente su alcance a todo el SV). Para un estudio comparado entre ambos adverbios, v. De Cesare (2007).

${ }^{22} \mathrm{La}$ ausencia de valores escalares en el focalizador español también puede explicarse por su origen etimológico a partir de una estructura comparativa (tan bien como) que establece una relación de igualdad entre ambos términos de la comparación (Sainz 2006b: 267). 
En italiano, funcionan como adverbios focalizadores aditivos escalares, además de anche y pure, addiritura, persino, perfino, y los menos frecuentes finanche, financo, fino que pertenecen a un registro escrito formal (literario según el Vocabolario Treccani) y el arcaísmo puranco ${ }^{23}$. Aunque en esta sede solo nos ocuparemos de los equivalentes más frecuentes, presentamos aquí algunos ejemplos de las otras tres formas (para puranco véase la n. 23):

(11) Bologna è una città ambigua, per molti emiliana, per qualcuno romagnola. Quaranta chilometri di portici, nel solo centro storico, segnano un'alternanza di luci e ombre unica al mondo. Le volte e le colonne disegnano nello spazio la doppiezza di una città divisa fra l'essere luccicante, ridanciana, esibizionista e trasgressiva e l'essere misteriosa, studiosa, finanche cospiratrice. (CORIS, MOND2008_10)

(12) L'inflazione in tutto l'Occidente non è più un problema. In Europa, anche Paesi con tradizioni di alta inflazione, come Italia, Spagna, Portogallo e financo la Grecia, si avviano a registrare i tassi più bassi degli ultimi trent'anni (questo mese l'inflazione italiana sarà con ogni probabilità inferiore al 2\%). (CORIS, STAMPAQuotidiani)

(13) Viveva le giornate fino in fondo, dall'abitudine alla sveglia mattutina fino a sera. Quando poteva si divertiva al gioco: scacchi, poker o écarté. Detestava perdere, soprattutto quando c'erano poste in palio. E per gli scacchi era pronto a ogni sfida: un anno era andato fino in Islanda per seguire il campionato del mondo tra Spasski e Fischer, negli anni più vicini aveva accettato di partecipare ai tornei casalinghi organizzati da Paolo Fresco, ex presidente della Fiat. (CORIS, MOND2008_10)24

La principal diferencia entre anche y pure, de un lado, y persino/perfino y addirittura, de otro, es que en los primeros el valor escalar no es intrínseco, es decir, no pertenece a la semántica nuclear del adverbio, que es puramente aditiva, sino que puede emerger en determinados contextos: se trata, por tanto, de un valor contextual. En este trabajo nos ocuparemos exclusivamente de los focalizadores con valor escalar intrínseco (Ricca 1999: 150).

${ }^{23} \mathrm{La}$ escasa frecuencia de esta forma en el italiano contemporáneo puede verificarse en el corpus manejado, que recoge solo 4 ocurrencias. Sin embargo, conserva en todos los casos el valor aditivo escalar: "Palazzo del Quirinale 31 dicembre 1991 Care Cittadine e cari Cittadini! è tradizione del nostro Paese che il Presidente della Repubblica, alla fine del vecchio ed alla vigilia del nuovo anno, rivolga un messaggio alla Nazione. Ma di tradizione pur sempre si tratta e non di legge imperativa: e ad essa, per seri motivi, è legittimo, anzi può essere, come nel caso presente, puranco doveroso, derogare". (CORIS, EPHEMOpuscoli). Mucho más frecuentes son finanche (174 ocurrencias) y financo (87). Para fino resulta difícil dar datos cuantitativos por las coincidencias con la preposición homónima.

${ }^{24}$ En este ejemplo, se advierte bien la diferencia entre la preposición fino, que precede complementos circunstanciales introducidos por las preposiciones $a$ e in (como en el primer enunciado: fino in fondo, fino a sera) y cuya presencia es obligatoria para la buena formación de la oración, y el adverbio focalizador fino que podría suprimirse: un anno era andato in Islanda. 
En tanto que conector, incluso puede ser considerado un conector textual aditivo 25 (Garrido Medina 1993, Cuartero Sánchez 2002). Los enfoques sintácticos explican en términos de alcance la diferencia entre las funciones de un focalizador y de un conector. En este último caso, su alcance no se limita a un elemento sintagmático o infrasintagmático sino que se extiende a toda una proposición, de modo que desaparecen las diferencias entre foco y plano secundario (background) en los que incluso articula el enunciado cuando funciona como adverbio focalizador. «The extension of the scope and the lack of common background reduce the focusing function of the adverbs but enhance their additive meaning, which is the basis of the linking function and the logical relation of addition» (De Cesare / Borreguero 2014).

El carácter aditivo de incluso le lleva a conectar dos enunciados que conducen a una misma conclusión, es decir, que están argumentativamente co-orientados. De hecho, en el marco de la Teoría de la Argumentación, incluso se considera un reforzador argumentativo que puede actuar en dos sentidos: introduciendo un segmento discursivo que aporta un argumento con mayor fuerza argumentativa que el precedente (uso reforzador) e introduciendo un segmento discursivo marginal al argumento principal (uso exceptivo) (cfr. García Negroni 1998).

Las diferencias en el alcance vienen marcadas habitualmente por cambios en la posición dentro del enunciado ${ }^{26}$. Así, cuando incluso desempeña una función

25 La relación existente entre los focalizadores aditivos y los conectores aditivos ha sido señalada por diversos autores (cf. König 1991; Mederos 1988: 224; Sabatini / Coletti 1997; Andorno 2000: 52-53; De Cesare / Borreguero 2014). De hecho, algunos autores los denominan "adverbios conjuntivos" para indicar esta función conectora en el nivel intraoracional, oracional y textual (Kovacci 1999: 769). Además, hay que señalar que cuando funcionan como conectores los focalizadores co-ocurren frecuentemente con conjunciones coordinantes (e incluso, o incluso), lo que refuerza aún más su valor aditivo. En el CORIS se registran 1676 ocurrencias de e persino (de las 9041 recogidas), 1274 de e perfino (de las 6440 recogidas), pero solo 812 de $e$ addirittura (de las 12.772 recogidas, si excluimo 8 caso con errores ortográficos). Menos frecuentes las combinaciones con la conjunción disyuntiva: 1770 ocurrencias de o addirittura, 130 de o persino y 72 de o perfino, lo que pone de manifiesto una cierta tendencia a utilizar el adverbio persino en las estructuras coordinadas conjuntivas y addirittura en las disyuntivas.

${ }^{26}$ Nótese que el concepto de alcance no es sinónimo del de foco, aunque el alcance y el foco pueden coincidir en algunos casos (Dimroth / Klein 1996). El alcance está formado por todos los elementos que caen bajo la influencia del focalizador, mientras que el foco es normalmente un único constituyente o un elemento intrasintagmático que recibe de forma más directa la acción del focalizador y constituye, por tanto, el elemento informativamente más relevante. «The focus of a particle can be defined as that string of expressions which is set off from the rest of the sentence by prosodic prominence and which is specifically affected semantically by the particle [...] It is, however, not only the focus that the contribution made by the particle to the meaning of a sentence depends on. Focus particles are also scope-bearing elements, so that their contribution to sentence meaning also depends on the scope they take within a sentence» (Köning 1993: 979). En la realización oral resulta relativamente fácil distinguir el foco del alcance porque el primero suele recibir mayor intensidad prosódica. Véase el ejemplo Hemos comprado incluso pasteles DE $N A T A$ en el que el alcance está formado por pasteles de nata pero el foco solo por de nata. En el texto escrito diferenciar ambos elementos resulta mucho más complejo y es imprescindible tener 
como focalizador suele ocupar una posición interna (a menos que focalice el primer constituyente) y prosódicamente (o gráficamente) está integrado en el enunciado. Sin embargo, cuando funciona como conector suele ocupar la posición inicial del enunciado y presenta un contorno prosódico independiente, separado por pausas (o por comas) del resto del enunciado. En esta posición y con esta función de conector textual, los equivalentes italianos más adecuados son inoltre, in più y, en algunos contextos, addirittura.

Lo que trataremos de mostrar aquí es que el Modelo de Basilea ofrece una base teórica más precisa para describir las distintas funciones discursivas de este elemento que las que ofrecen otros enfoques basados únicamente en los cambios en la posición enunciativa y, por consiguiente, en el alcance o cantidad y tipo de elementos afectados por la semántica de incluso, lo que, por otra parte, permite determinar con mayor precisión cuáles son sus equivalentes italianos más adecuados en cada caso.

\section{FOCALIZADORES ADITIVOS ESCALARES EN LA UNIDAD DE NÚCLEO}

Cuando el adverbio focalizador y el elemento focalizado se encuentran en la Unidad de Núcleo -y esta constituye una estructura predicativa completa-, el focalizador tiene una amplia libertad de movimiento dentro del Enunciado. De hecho, puede ocupar numerosas posiciones tanto en el bloque remático de la Unidad de Núcleo (14) como en el bloque temático (15), aunque lo habitual es que el focalizador preceda al elemento focalizado (que aparece subrayado en los ejemplos) o insertado en este cuando presenta una cierta complejidad, como en el caso de los predicados verbales perifrásticos (v. 14). Son raros, sin embargo, los casos en que aparece pospuesto al foco (pero véase López Serena / Loureda 2013). Por otra parte, como es característico de los adverbios de foco, pueden combinarse con elementos sintagmáticos de todas las categorías (aunque son especialmente frecuentes los SSNN y los SSVV focalizados) y en el desempeño de cualquier funcion sintáctica.

(14) El incidente se produjo en la ciudad iraní de Hamadán, a unos doscientos kilómetros al suroeste de Teherán. Los agresores la emprendieron a golpes con todos los presentes en la asamblea. Entre los heridos se encuentran el diputado reformista Hosein Loghmanian, el líder estudiantil Said Razavi Fagih y Hosein Mojahed, máximo responsable de la agrupación local del llamado Frente Participativo, la organización democrática iraní más importante. // Mojahed tuvo que ser incluso hospitalizado con serios traumatismos en cara y un brazo./Núcleo // E (El Diario Vasco, 23/01/2004)

(15) Pero pasó lo mismo en Francia, donde a partir del dominio abusón de Rossini, Bellini, Donizetti y después Verdi, se desarrolló la ópera cómica:

en cuenta la información ofrecida por el cotexto, pero incluso así los casos de indeterminación no son infrecuentes. 
"Los compositores franceses se refugian en ese campo. // Incluso los más grandes de la ópera francesa son, $/$ Núcleo- sobre todo, $/{ }^{\text {Apéndice }}$ autores de ópera cómica"./-Núcleo $/ /_{\mathrm{E}}$ (El País, 14/06/2004)

Esta situación es perfectamente simétrica en el caso de los adverbios italianos persino/perfino ${ }^{27}$, que, sin embargo, parecen admitir con más facilidad la anteposición del foco, como se ve en (18).

(16) Mordent è sbarcato nello stabile occupato, chiamato dai poliziotti di Bicêtre, e là dentro era l'inferno. Non ha bisogno che le descriva la scena. // C'erano persino dei ragazzini che mangiavano scatolette per cani. /Núcleo $/ /_{\mathrm{E}}$ (CORIS, MOND2008_10)

(17) Si tratta di alfabeti che utilizzano caratteri differenti e hanno spesso anche regole di composizione molto diverse, cosa che comporta l'utilizzo di tutta una serie di simboli di punteggiatura e di variazione fonetica che non hanno un corrispondente negli alfabeti occidentali. // Persino gli spazi bianchi non sono gli stessi./Núcleo // (CORIS, MISCRiviste)

(18) Non conoscono i sordi, loro. Ogni giornalista mi dà l'impressione di scoprire la nostra esistenza. // Sono gentili, adorabili, appassionati, attenti, ammirati persino. Nícleo $/ /_{\mathrm{E}}$ (NARRATTrVaria) ${ }^{28}$

Sin embargo, en el caso de addirittura la situación es más compleja. Se encuentran ejemplos en los que el elemento focalizado es un SN (19), un SAdj (20), un SAdv (21) y un SPrep (22) y en este punto no parece presentar diferencias con persino/perfino, frente a lo que afirma Visconti (2004: 446).

(19) Sospira: «Ho dimostrato una capacità di sopportazione straordinaria, ma adesso... Comunque ognuno fa la sua strada». Una strada che s'incrocia, un'altra volta: «Durante le Regionali, ci fu il tormentone delle firme - riassume per lui il collega Altero Matteoli -. // Ora,/ addirittura lo spionaggio!/Núcleo// Oltre al danno, ecco la beffa». (CORIS, MOND2005_07)

(20) Siamo ben lontani dalle lettere di Hugo alla fidanzata , eccezion fatta per loceanico carteggio con la sua amante in titolo Juliette Drouet. // Anche se oggi con il telefono un'impresa del genere sarebbe addirittura impensabile/ ${ }^{\mathrm{Nu}-}$ cleo $/ /_{\mathrm{E}^{*}}$ (CORIS, STAMPAQuotidiani)

(21) Lerrore è umano anche in questo senso: // gli animali non si sbagliano che di rado o addirittura mai,/Núcleo ad eccezione dei più intelligenti.// E (CORIS, MISCOpuscoli)

27 Dado que en el caso de persino y perfino se trata de dos variantes gráficas del mismo adverbio, nos limitaremos a dar ejemplos de uno u otro caso para no alargar innecesariamente este estudio.

${ }^{28}$ El ejemplo está extraído de un texto literario traducido, pero en el corpus se encuentran otros ejemplos, la mayoría en textos literarios y tras el último término de una enumeración: "Il generale Garibaldi era cuoco geniale e ottimo fattore. Per tutto quel febbraio di flagelli pensò lui ad ogni cosa, cucinava, accudiva il pollame, pensava al bucato persino." (CORIS, NARRATRomanzi). 
(22) // Il Granducato ha reagito prontamente affermando che/Núcle- in base ai suoi calcoli/ il proprio contributo netto al bilancio comunitario lo porrebbe addirittura al primo posto nell'Ue tra tutti i Paesi che danno più di quanto ricevano./-Núcleo// $\mathrm{E}$ (CORIS, STAMPAQuotidiani)

Pero en la mayoría de los ejemplos el alcance del adverbio addirittura se extiende a todo el SV (23) o incluso a todo el enunciado, aunque, como veremos más abajo, en estos casos la realización parentética (con frecuencia marcada mediante comas en la escritura) nos indica que el adverbio no está integrado en la Unidad de Núcleo.

(23) Come si dovrebbe comportare un imprenditore in questa situazione? Se è molto prudente, non sarebbe un imprenditore vero. // Se è invece aperto alle possibilità che si aprono da una crisi di questo genere/ potrebbe addirittura approfittare della situazione./Núcleo // (CORIS, STAMPAQuotidiani)

No obstante, la principal diferencia con otros adverbios focalizadores escalares reside en que addirittura evita la focalización de un constituyente en posición inicial de enunciado porque su tendencia a extender su alcance a todo el enunciado induciría a equívoco. Es lo que ocurre en (24),

(24) È un'indecenza che uno dei più bei giardini di Torino autentico salotto della città, sia trasformato in area riservata a cani. // Qui/ addirittura/ i quattrozampe non dovrebbero essere lasciati liberi di scorrazzare come fossero i padroni delle aiuole, ma come minimo essere tenuti al guinzaglio./Núcleo//E (CORIS, STAMPAQuotidiani)

donde el foco del adverbio no son i quattrozampe (ya que no es posible pensar en una alternativa - ¿quién más no debería pasearse sin correa? - ) sino todo el enunciado: no solo el parque no debería ser un espacio reservado a los perros, sino que ni siquiera se les debiera permitir pasear sueltos. La interpretación correcta nos lleva, por tanto, a no considerar addirittura un focalizador integrado en el núcleo, sino un elemento prosódicamente independiente.

Otro argumento que viene a reforzar esta hipótesis es que los adverbios aditivos escalares son sensibles a la polaridad afirmativa o negativa del enunciado, es decir, tanto el español incluso cuando funciona como focalizador, como persino / perfino y addirittura, cuando funciona como focalizador, aparecen en enunciados con polaridad afirmativa. En caso contrario son sustituidos por focalizadores escalares restrictivos: ni siquiera, neanche, nemmeno, neppure.

(25) Sarei curioso di sapere a quanto ammonta il deficit dei trasporti pubblici a Palermo, visto che in quella città nessuno timbra il biglietto. Viene spontaneo il suggerimento di abolirne la vendita ai capolinea, tenuto conto che con 
gli scarsi proventi l'Azienda municipale non recupera neanche i soldi per lo stipendio ai vari preposti. (CORIS, EphemLettere)

$\left(25^{\prime}\right)^{*}$ non recupera perfinoladdirittura i soldi per lo stipendio ai vari preposti

De modo que si en (24) el elemento focalizado fuese $i$ quattrozampe el focalizador tendría que ser un escalar restrictivo portador de la polaridad negativa del enunciado:

(24') Qui/ nemmeno i quattrozampe dovrebbero essere lasciati liberi di scorrazzare come fossero i padroni delle aiuole, ma come minimo essere tenuti al guinzaglio./Núcleo

Otros ejemplos en este sentido serían (26) y (27), donde el alcance de addirittura se extiende a todo el enunciado y no funciona, por tanto, como un focalizador:

(26) Per valutare lo stato dei diritti umani invece gli analisti osservano il prezzo a cui viene venduto il kalashnikov. Meno costoso è il mitra, più i diritti umani sono violati, lo Stato di diritto è in cancrena, l'ossatura degli equilibri sociali è marcia e in disfacimento. Nell'ovest dell'Africa può arrivare a 50 dollari. Addirittura in Yemen è possibile rintracciare AK-47 usati di seconda e terza mano anche a 6 dollari. (CORIS, MON2005_07)

(27) Perché, per esempio, gli avvocati sono presentati solo come comprimari di Tangentopoli o non vengono chiamati in causa? Marco De Luca, difensore di Raul Gardini, parla per pochi secondi interpellato semplicemente sullo stato d'animo del suo cliente prima del suicidio. Addirittura Giuseppe Lucibello, l'avvocato amico di Di Pietro e del finanziere Francesco Pacini Battaglia, è ignorato. (CORIS, STAMPAPeriodici)

De cualquier forma, en el caso de addirittura, conviene hablar de tendencia a no ocupar la posición inicial focalizando un constituyente sintagmático más que de imposibilidad, ya que se encuentran algunos casos en los que addirittura mantiene su función de focalizador en esa posición (28) en analogía a perfino / persino (29):

(28) La pista infatti si è asciugata grazie al sole seguito alla pioggia di stamane, ma ancora non è perfettamente gommata. Tempi relativi, dunque, ma sufficienti a dimostrare una supremazia Ferrari in una giornata in cui la Williams - Bmw di Ralf Schumacher si è fermata a 733 millesimi, mentre Hakkinen è solo quarto e distanziato di 1"245, davanti a Olivier Panis su Bar - Honda e Kimi Raikkonen su Sauber. // / Addirittura undicesimo David Coulthard,/Núcleo a 2"036, peraltro fermato al $24^{\circ}$ giro (Schumi ne ha fatti 44) da una uscita alle Acque Minerali con motore spento.//E (CORIS, MON2001_04)

(29) "Giochi senza frontiere" si intitola un articolo di Liberation sul successo dei videogiochi. // / Perfino la Microsoft si è gettata a capofitto in questo 
business,/Núcleo che vale 20 miliardi di euro l'anno (quasi 40 mila miliardi di lire, ndr) e dà lavoro a 65 mila addetti. // E (CORIS, STAMPAQuotidiani)

La diferencia entre el alcance perfino/persino, de una parte, y addirittura, de otra, se debe, según Visconti (2004: 446 y passim; 2005), a la diversa categoría gramatical a la que pertenecían en origen estos elementos. Mientras perfino / persino (formados a partir de PER + FINIS) fueron en un principio preposiciones que indicaban direccionalidad hacia un límite temporal o espacial y cuyo proceso de gramaticalización parece paralelo al de la preposición española hasta (aunque este extremo requiere una investigación en profundidad $)^{29}$, addirittura proviene de una locución adverbial (a dirittura) con el significado de 'sin dilación, directamente' ${ }^{30}$. Por tanto, esta diferencia en el origen gramatical de ambos focalizadores - preposicional y adverbial, respectivamente- es lo que explicaría las diferencias en el alcance y su distinto comportamiento sintáctico, así como la posibilidad de addirittura de funcionar como conector textual, como veremos más abajo.

[...] perfino still retains selectional properties inherited form its origin as a prepositional modifier; thus, it has a more restrained distribution in comparison

${ }^{29}$ El proceso de gramaticalización (o de subjetivización, en la concepción de Visconti 2004, 2005) de perfino/persino como focalizador escalar es mucho más temprano que el de addirittura y desde el s. XV ya se encuentran ejemplos en los que el complemento espacial o temporal es presentado como extremo o inesperado (perfino aipiù segreti luoghi): "L'ipotesi è che la componente scalare abbia finito per convenzionalizzarsi grazie alla frequente associazione del lessema con contesti in cui essa è espressa da altri mezzi linguistici [...] Il valore scalare si sviluppa come convenzionalizzazione della componente valutativa / soggettiva del parlante / scrivente, espressa prima dai contesti, poi gradatamente assorbita dai lessemi stessi» (Visconti 2004: 456, 461). Las primeras ocurrencias en las que ha desparecido el valor espacial y temporal son del s. XVI, donde perfino ya no precede a un SPrep sino a un SN (Perfino una catena d'oro di sesanta scudi), si bien estos valores persisten hasta bien entrado el s. XIX.

30 Siguiendo a Visconti (2004: 451-454), este primitivo uso adverbial, que tenía en un principio únicamente valor espacial (andò addirittura alla rocca Sansimone), aparece a partir del s. XVI en contextos donde adquiere valores temporales (Intanto ci furono avisi di Francia, come il re ne veiva a dirittura in Italia con animo prontissimo di salvare noi). Posteriormente, la ocurrencia de este adverbio en un contexto donde se enumeran una serie de alternativas relativas al momento propicio para una acción, que son descartadas, mientras se presenta como alternativa válida, pero extrema o inesperada, aquella precedida por addirittura, habría dado lugar, según esta autora, a la emergencia del valor escalar al "absorber" el adverbio el componente evaluativo y la función textual de marcar la alternativa (poiché il nuncio Grimalid [...] non si prese cura alcuna di ciò che il papa co" suoi monaci sentisse di me e della mia opera; né con l'Imperatore o con altri ministri passò contro di me doglianze. Ma i mali uffici venivano a dirittura da Roma). Ejemplos en los que la transición es aún más clara, porque el adverbio aparece junto a verbos que no indican movimiento, se encuentran en el s. XVII (Quanti arrivano a questa locanda, tutti di me s'innamorano, tutti mi fanno i casca morti; e tanti, e tanti mi esibiscono di sposarmi a dirittura). Para un estudio en profundidad de la evolución histórica de estos focalizadores y para la fuente de los ejemplos citados en esta nota y en la anterior, cf. Visconti $(2004,2005)$. 
con addirittura, which, unlike perfino, can be used in Present Day Italian with sentential scope $[\ldots]$ and as an interjection (Visconti 2005: 246).

Por otra parte, es posible detectar una diferencia semántica entre los focalizadores italianos que concierne a su valor aditivo. En opinión de Visconti (2005: 245-246), el significado léxico de origen de estos elementos condiciona sus valores discursivos actuales, de modo que el valor aditivo está siempre presente en perfino/persino -que es inherentemente culminativo- mientras que no lo está en addirittura -que emerge de una construcción preposicional con valor direccional- como se aprecia en el hecho de que este último puede combinarse con focalizadores aditivos como anche:

(30) Un altro atleta, Angelo Jacopucci, aveva perso la vita dopo un match sostenuto e perso per k.o. a Bellaria contro l'inglese Alan Minter il 19 luglio 1978. $\mathrm{E}$ anche in quelloccasione la coda giudiziaria fu pesantissima: furono incriminati il manager di Jacopucci, Rocco Agostino, il medico della riunione, Ezio Pimpinelli, e addirittura anche l'arbitro dell'incontro, il francese Baldeyron. (CORIS, MISCVolumi)

$\left(30^{\prime}\right)$ *e perfino anche l'arbitro dell'incontro

En otros Enunciados, la Unidad de Núcleo no está formada por una estructura predicativa, sino por un único constituyente sintagmático desgajado de otro enunciado, un recurso expresivo bastante habitual en el lenguaje periodístico contemporáneo. En este caso, al tratarse de la focalización de un único elemento (sin ambigüedades posibles respecto a su alcance) se comportan como equivalentes de incluso (30) tanto perfino/persino (31) como addirittura (32):

(30) Nacida cerca de La Habana hace 64 años, ha pasado dos terceras partes de su vida en el exilio y se confiesa "republicana de la cabeza a los pies", partido al que vota desde que logró la ciudadanía. Las medidas contra Castro le parecen "muy bien". Del presidente Bush le gusta todo. // / ¿Incluso la guerra?/Núcleo // "Incluso la guerra"./Núcleo/// (CREA, $A B C, 03 / 11 / 2004)$.

(31) Ma Lippi, pur contrariato, non si è sottratto alla dialettica; Louis Van Gaal, invece, come Mike Bongiorno nei quiz, ha comunicato agli italiani scesi ad Asti e attardatisi a conversare con Litmanen: "Il vostro tempo è scaduto". Stasera al Delle Alpi, 63 mila persone, cinque miliardi e mezzo di incasso, un miliardo e mezzo per l'Ajax se fa il miracolo, potrebbe terminare il suo. // / Perfino teatralmente./Núcleo // (CORIS, STAMPAQuotidiani)

(32) Marco Minniti - numero due del Pds - non è mai riuscito a mandarla giù la polemica scatenata da Bertinotti su Di Pietro candidato senatore ulivista del Mugello. Adesso, dopo l'arrivo di Kojak-Curzi la cosa gli sta proprio sul gozzo. Perché - giura - "le premesse su cui si regge tutta la polemica sono false". // /Addirittura false?/Núcleo // E (CORIS, STAMPAQuotidiani) 
En estos ejemplos, incluso forma parte de la unidad de Núcleo junto a otro material lingüístico, pero en ocasiones puede saturar por sí solo el Núcleo, cuando constituye un turno de habla en conversaciones coloquiales, aunque su frecuencia es menor que la de otros adverbios focalizadores. Además de realizaciones ecoicas que tienen un evidente carácter enfático, como en (33-34):

(33) Joder! Os vamos a dar, incluso, además de todo, a pesar de, una pista. Vamos a dar ¿Una pista? Sí. Bueno, pues, no sé. Vamos a darla, venga. Toma toma una pista. Ahora mismo. Pues no sé. Al el teléfono al que tengo que llamar, por ejemplo, eso es una buena pista para ganarme el premio. Venga, sí, es una buena pista. Además, podéis llamar a a ¿cómo es? // Al A cobro

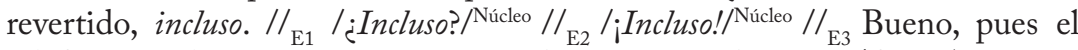
teléfono es el siguiente: cinco-cinco-dos-uno-seis-dos-tres. (CREA, Canela en Rama, Punto de mira, Radio Vallecas B, 12/06/1991)

(34) Ieri, parlando ai Club di Forza Italia, ha di fatto spiegato la genesi della «frattura». Si è «rotto» perché «ci sono amici dei comunisti in ogni partito».// /Persino nei partiti amici?/Núcleo // E1 Persino./Núcleo// E2 (CORIS, MON2001_04)

este focalizador puede aparecer saturando un núcleo en otros contextos, aunque son muy poco frecuentes ${ }^{31}$. En estos casos, el foco no está expreso y debe recuperarse inferencialmente a partir del cotexto o del contexto. Como hemos señalado en otro lugar (De Cesare / Borreguero 2014), el focalizador tiene aquí un uso holofrástico y se emplea como respuesta afirmativa enfática a interrogativas totales (35) o como confirmación de lo aseverado por el interlocutor (36) 32 :

(35) A: A mí me encantan las verduras.

$\mathrm{B}:$ ¿Y las acelgas?

A: /;Incluso!/Núcleo

(36) A: Pero, Pepe, ¡is has hecho ya la cena! ¡Y has recogido la cocina! B: /iIncluso! /Núcleo ¡Para que veas!

Esta posición del focalizador saturando la Unidad de Núcleo se documenta en italiano, pero únicamente en el caso de addirittura, lo que pone de nuevo de relieve el diferente comportamiento textual de este focalizador frente a la pareja persinolperfino ${ }^{33}$. Son especialmente frecuentes las ocurrencias en Enunciados interrogativos (37) o exclamativos (38), pero no solo (39). Todos los ejemplos

${ }^{31}$ De hecho, no hemos encontrado ningún caso ni en los datos orales del Corpus CREA ni tampoco en los del Corpus Val.Es.Co.

${ }^{32}$ Los ejemplos están tomados de Ferrari/Borreguero (2015). En este caso se trata excepcionalmente de ejemplos inventados, porque, como hemos dicho, no hemos encontrado ninguna ocurrencia de incluso saturando la Unidad de Núcleo en nuestro corpus, pero nuestra competencia de hablantes nativos nos permite asegurar con convicción que este tipo de Enunciados son posibles.

${ }^{33}$ Téngase en cuenta que el ejemplo de (34) es un caso de enunciado ecoico y, por tanto, con características particulares respecto de otros casos de saturación de Núcleo. 
encontrados pertenecen a textos en los que se reproducen diálogos reales o ficticios, lo que corrobora el carácter oral de este tipo de Enunciados.

(37) Sarà commissario con pieni poteri e risponderà direttamente a Fini. Ł̀ così? "Sì, il mandato è quello. Nei giorni scorsi Gianfranco mi ha chiesto di tornare al partito. Gli ho chiesto di considerare la mia disponibilità un atto di generosità". // /Addirittura?/Núcleo // "Beh, la partita è delicata, delicatissima. Roma è per Alleanza nazionale quello che Bologna è per il Pds [...]”. (CORIS, STAMPAQuotidiani)

$\left(37^{\prime}\right)^{*}$ Gli ho chiesto di considerare la mia disponibilità un atto di generosità. Perfino?

(38) Ancora oggi si strepita sulle "migliaia di miliardi" (scarsi centoquaranta miliardi, in euro) della Cassa per il Mezzogiorno, in quarant'anni. Costituivano lo 0,5 per cento del prodotto interno lordo. Altri conteggi spingono la cifra allo 0,7.///Addirittura!/Núcleo // $\mathrm{E}$ È tanto? È quasi niente: fra un centocinquantesimo e un duecentesimo della ricchezza annua dell'Italia; (CORIS,MON2008_10) ${ }^{34}$ (39) Nel dialogo tra la Lipari e il suo amico registrato dagli agenti della Digos non compare mai il nome del "procuratore". Parlando del magistrato, la donna dice all'interlocutore: "Però non è che gli ho detto guardi che lei non ha il diritto io la denuncio... perché questo diceva "sputtano lei , sputtano suo padre eccetera" [sic]. L’amico replica alla Lipari: // /“Addirittura"/Núcleo //E E lei specifica: "Eh sì... proprio questa è l'espressione precisa". (CORIS, STAMPAQuotidiani)

$\left(39^{\prime}\right)$ *L'amico replica alla Lipari: "Perfino"

Para finalizar con la casuística de los adverbios focalizadores en la Unidad de Núcleo, queremos llamar la atención sobre una posición que prefigura en cierta forma la función que adquieren estas unidades léxicas cuando se sitúan en la Unidad de Marco, tal y como veremos a continuación. Cuando el focalizador aparece en contigüidad con el verbo, normalmente en posición preverbal pero en ocasiones también en la postverbal, extiende su alcance a todo el enunciado de tal modo que no es posible identificar con claridad un único constituyente como foco. Esta posición favorece así la prominencia del valor aditivo (en detrimento de la función focalizadora, que sin embargo no desaparece), valor reforzado por el propio contenido semántico del Enunciado en relación con los Enunciados precedentes. En (40) la mala situación del sector se refleja en tres hechos que se orden en una escala pragmática: se paralizaron proyectos, otros no llegaron a ponerse en marcha, algunos proveedores quebraron.

(40) El año pasado fue duro para el sector del e-learning (formación a través de Internet) en España. La mala evolución de la economía hizo mella en los

34 En estos casos, el carácter enfático puede sustituirse por una expresión de sorpresa del tipo ¡No me digas! ¿ Qué me dices? que es el equivalente que aparece en algunos diccionarios, cf. Arquès / Padoan 2012: s.v. addirittura 
presupuestos que las grandes empresas destinan a formar a sus empleados. Se paralizaron proyectos y otros no llegaron a ponerse en marcha. // / Incluso quebraron algunos proveedores./Núcleo // (CREA, El País, 16/02/2003)

En esta posición nos encontramos de nuevo con una diferencia entre addirittura y perfino/persino, pues mientras el primero se comporta de manera muy semejante a incluso en estos casos, tanto la posición preverbal como la extensión del alcance a todo el enunciado parecen bloqueados para perfino / persino como se ve en $\left(41^{\prime}\right)^{35}$ :

(41) Non aveva intenzione di fotografarli a tutti i costi. Dopo i primi scatti, depose la macchina fotografica e cominciò a giocare. Capii che non avrebbe guadagnato una lira come reporter, il suo occhio non era di quelli che restano incollati alla macchina morbosi e ciechi. Lo vidi rinunciare agli scatti migliori in favore di altri che semplicemente divertivano i bambini. // /Addirittura mise la macchina fotografica al collo di uno di loro e gli lasciò sputtanare un intero rullino/Núcleo $/ / \mathrm{E}_{\mathrm{E}}$. Tornammo con un servizio misero, invendibile. (CORIS, MON2008_10)

$\left(41^{\prime}\right)^{*}$ Perfino mise la macchina fotografica al collo di uno di loro e gli lasciò sputtanare un intero rullino

Otro factor que apoya la naturaleza intermedia entre focalizador y conector en esta posición es el hecho de que, mientras resulta extremadamente raro que el focalizador no ocupe una posición adyacente a los elementos que componen su alcance cuando desempeña una función estrictamente focalizadora, no es imposible que en estos casos esté separado por medio de un inciso delos elementos léxicos bajo su alcance. Se refuerza así el significado aditivo que alcanzará su máxima expresión cuando desempeña una función predominantemente conectora, como veremos más abajo.

35 Esta restricción es similar a la de anche que, al contrario de lo que ocurre con el español también, tampoco puede extender su alcance al conjunto del enunciado ni funcionar, por tanto, como un conector textual. Esta limitación funcional se refleja en la imposibilidad de ocupar la posición inicial de enunciado precediendo a un verbo. Así en un texto como (a) no sería posible anteponer el adverbio al verbo (a'), un error típico, por otra parte, de los aprendices hispanófonos de italiano (Borreguero 2012):

(a) Percorriamo i numerosi sterrati incontrando parecchi animali fino a quasi l'orario di chiusura e concludiamo la visita nel Centenary Centre dove vengono tenuti gli animali (per la maggior parte rinoceronti e bufali al momento) in attesa di trasferimento in altri parchi africani. Visitiamo anche il ricco centro di artigianato dove si può trovare una vasta scelta di manufatti tipici. (CORIS, MON2004_01)

(b) Anchevisitiamo il ricco centro di artigianato dove si può trovare una vasta scelta di manufatti tipici.

Sobre las diferencias entre anche y también, cf. Sainz (2006a), Borreguero (2011) y De Cesare/ Borreguero (2014). 
(42) Desde que José María Aznar se hizo cargo del PP, sus correligionarios catalanes se encuentran en una permanente rencilla sorda que ni siquiera las fotografías victoriosas de las últimas elecciones han conseguido mitigar. La elaboración de las listas acostumbra a ser un auténtico encaje de bolillos. Las cartas de denuncia y de conspiraciones van y vienen de la sede de la calle Urgell a la sede de la calle Génova. // /A veces,/Marco incluso/Núcleo- // -y Javier Arenas lo recuerda bien-//Inciso ${ }_{\mathrm{E} 2}$ ha habido que mandar a una institutriz de Madrid para que pusiera orden entre los popes populares./-Núcleo $/ / \mathrm{E} \mathrm{El}$ factor humano todavía es más importante que el factor político. (CREA, La Vanguardia, 30/06/1995)

(43) Sono io il presidente del partito, dice Fini, perciò mi assumo io la responsabilità per come è andata. L' esecutivo gli ha rinnovato la fiducia, però è troppo facile farsi confermare dai propri collaboratori, "non mi basta". Il leader vuole discutere della batosta con la direzione.// Addirittura,/Núcleo- in un primo momento, ${ }^{A p e ́ n d i c e ~ a v e v a ~ p e n s a t o ~ d i ~ c o n v o c a r e ~ p e r ~ s a b a t o ~ a n c h e ~ l ' a s s e m b l e a ~}$ nazionale./-Núcleo // E CORIS, STAMPAQuotidiani)

Que el adverbio pertenece a la Unidad de Núcleo -y no a la de Marco, como podría pensarse por la situación- resulta evidente cuando se suprime el Apéndice (43) o Inciso (42) 36 incrustado en el Núcleo, ya que en ese caso no hay pausa tras el focalizador en la realización oral y, por tanto, desaparecía la coma en el texto escrito, a diferencia de lo que ocurre en la Unidad de Marco, que pasamos a examinar a continuación.

\section{FOCALIZADORES ADITIVOS ESCALARES EN LA UNIDAD DE MARCO}

Cuando incluso aparece en la Unidad de Marco cambian tanto su alcance y su función como su valor pragmático. Dado que una de las principales funciones de la Unidad de Marco es la de servir de enlace con el Enunciado o los Enunciados precedentes, las partículas discursivas situadas en este Unidad, especialmente si la saturan por completo, tienden a adquirir funciones claramente conectivas.

Sin embargo, existe una diferencia importante entre a) las ocurrencias en las que incluso va acompañado de otros elementos léxicos en la Unidad de Marco y b) aquellas en que satura dicha Unidad.

a) En las primeras prevalece la función focalizadora, que limita su alcance a los elementos contenidos en el Marco, pero incluso adquiere ya un valor muy próximo al de conector aditivo que deriva del hecho de que con frecuencia la información contenida en la Unidad de Marco mantiene su vigencia para las

36 Según el modelo de Basilea, a diferencia del Apéndice que es una Unidad Informativa dentro del Enunciado, el Inciso tiene entidad de Enunciado, pues posee autonomía ilocutiva y semántica (Cignetti 2011). Aunque no hay un uso coherente de los signos de puntación, el Inciso suele aparecer entre guiones (rayas según la última ortografía de la RAE) o bien entre paréntesis, mientras que el Apéndice suele hacerlo entre comas. Pero, como decimos, este uso no es consistente, de modo que es necesario analizar la función textual y la contribución pragmáticodiscursiva para poder dilucidar si se trata en cada caso de un Apéndice o de un Inciso. 
sucesivas Unidades de Núcleo que componen el Enunciado ${ }^{37}$. Así, en (44), incluso focaliza un complemento circunstancial de lugar que activa un marco semántico, es decir, que constituye una información espacial que mantiene su validez para las acciones sucesivas protagonizadas por los coches y las personas respectivamente:

(44) El Estado judío quedó paralizado ayer durante dos minutos mientras se escuchaba en todo el país una sirena. Cerca de cinco millones de personas recordaron en silencio a los seis millones de víctimas del Holocausto nazi de la Segunda Guerra Mundial. // /Incluso en las calles y en las autopistas del país,/Marco los coches se detenían/Foco1 y las personas salían de sus vehículos/Foco2./Núcleo // Era el cincuenta aniversario de la liberación de los campos de concentración. (CREA, La Vanguardia, 28/04/1995)

Nótese, sin embargo, que la acción de incluso no se limita a focalizar el elemento inicial, sino que todo el enunciado que introduce (con su doble Foco) constituye un argumento que viene a reforzar la tesis inicial de que $e l$ Estado judio quedó paralizado ayer durante dos minutos. En efecto, que los coches se detenian y las personas salian de sus vehiculos constituyen dos hechos que ponen de manifiesto la parálisis del país. No se pierde el valor escalar, de modo que estos hechos se sitúan en un lugar superior en la escala argumentativa respecto del descrito en el enunciado inmediatamente anterior: cerca de cinco millones de personas recordaron en silencio a los seis millones de victimas del Holocausto. La detención de los vehículos en calles y autopistas es un acontecimiento menos esperable y a la vez un argumento irrefutable de la tesis inicial ${ }^{38}$. El valor de conexión aditiva se observa más claramente porque se podría trasladar el complemento circunstancial a la posición final de enunciado sin que variara la función discursiva de incluso:

(44') Incluso,/Marco los coches se detenían y las personas salían de sus vehículos en las calles y en las autopistas.

En estos casos, cualquiera de los aditivos escalares italianos de los que nos ocupamos puede considerarse equivalente de incluso. Así en (45) perfino focaliza un complemento de lugar (in Vaticano) que constituye el marco espacial válido para el enunciado en el que se encuentra y para el siguiente. Del mismo modo, en (46) addirittura focaliza un complemento temporal (in un caso = una

37 Incluso podríamos decir que en determinadas ocurrencias textuales la función de conexión aditiva y de focalización se superponen en el llamado fenómeno de estratificación (layering, cf. Hopper/Traugot 1993) que ha sido bien descrito por la teoría de la gramaticalización.

38 En palabras de Fuentes (2009: s. v. incluso), «añade un enunciado coorientado a los anteriores, pero superior en la escala argumentativa y no esperado». 
volta) que constituye el marco en el que se ubican las acciones descritas en los Enunciados 1 y 2.

(45) Perfino in Vaticano/Marco parlano solo di calci./Núcleo // $\mathrm{E} 1$ Stamattina ho sentito che pregavano perché non crolli l'Olimpico./Núcleo // E2 (CORIS, MISCVolumi)

(46) $\grave{E}$ indubbio che la morte di Versace sia stata firmata dallo stile del filippino: ha lasciato sotto la macchina anche gli abiti che indossava quando ha sparato e con i quali si è deliberatamente fatto vedere e descrivere dai presenti. Pantaloni corti scuri, zainetto, maglietta. Ha abbondato in tracce. Ha voluto farsi riconoscere. Ma in passato non aveva fatto così. // / Addirittura in un caso,/Marco quello del miliardario Lee Miglin,/Apéndicel l'agente immobiliare di Chicago/Apendice2 //(un distinto signore di 72 anni, sposato, filantropo e noto per la sua generosità)/Apéndice3, ha combinato le cose in modo da lasciare dietro di sè una scia di dubbi./Núcleo // ${ }_{E 1}$ Certo,/Marco si è portato via la sua macchina, ma nessuno ha potuto metterlo direttamente in relazione con l'assassino./Núdleo // E2 (CORIS, STAMPAQuotidini)

En otros casos el alcance del focalizador engloba toda la subordinada, y de nuevo la función focalizadora pierde intensidad (es difícil identificar un elemento focal) respecto de la función de conexión aditiva. En esta posición enunciativa no es infrecuente que a este valor aditivo se superponga un matiz concesivo (Flamenco 1999, Montolío 1999, NGLE 2009, 47.2n, ñ y 47.12e) ${ }^{39}$ que también aparece en los Marcos precedidos por perfino / persino (Schiemann 2009: 218-220) pero nunca con addirittura. En efecto, con esta estructura se indica que las aseveraciones de los enunciados anteriores mantienen su validez a pesar de una situación o condición que se presenta como adversa: todos los atentados perturban la paz incluso los frustrados (47), la cesión de la colonia de Ariel a los palestinos nunca se ha planteado ni siquiera en la época en que se cedió el 97\% de Cisjordania (48):

(47) La necesidad de un mínimo de tranquilidad y de seguridad hace que los atentados frustrados se diluyan en el alivio general. Los propios medios de comunicación empleamos la coletilla "no ha causado daños personales" con cierta ligereza. Pero los atentados frustrados nunca son atentados fallidos. // /Incluso cuando la bomba queda sin explotar/Marco hay una onda expansiva que perturba la paz de sus víctimas, el miedo altera su ánimo y una terrible desazón se apodera de su alegría por seguir vivos./ ${ }^{\text {Núcleo } / / \mathrm{E}}$ Cada bomba que falla supone que decenas de personas tiemblan pensando que les podía haber tocado a ellas. (CREA, El Diario Vasco, 11/01/2001)

39 «Conviene insistir en que para obtenerse una interpretación concesiva condicional, el foco de estas partículas debe ser toda la proposición subordinada y no sólo un elemento de la misma» (Flamenco 1999: 3844). 
(48) Non pensate che, con un accordo di pace, il governo israeliano sgombererà alcuni insediamenti in Cisgiordania, proprio come ha fatto nel 2005 con le colonie di Gaza? Non possiamo parlare per gli altri insediamenti, ma non c'è alcun dubbio che Ariel è qui per restare. Questo è un posto strategico per l'industria israeliana e anche per la sicurezza: il 99 per cento degli israeliani sanno che Ariel è necessario per ostacolare un attacco dai Paesi arabi confinanti. // / Persino quando c'era Ehud Barak al governo,/Marco che offrì ai palestinesi il 97 per cento della Cisgiordania più alcuni territori israeliani come compensazione,/Apéndice del Marco cedere Ariel era fuori discussione./Núcleo $/ /_{\mathrm{E}}$ (CORIS, MON2008_10)

Como se ve en estos ejemplos, cuando los focalizadores aditivos escalares preceden a estructuras condicionales (con prótasis introducidas por si) $s e^{40}$ o cuando/quando, o por sin/senza + infinitivo o por gerundio), adquieren normalmente un valor concesivo ${ }^{41}$. En estos casos, el conector puede reemplazarse, con los ajustes sintácticos oportunos, por un conector concesivo pero sin abandonar su valor escalar que suele indicar una situación extrema o poco esperada ${ }^{42}$. Veamos el siguiente ejemplo:

(49) Alla fine la scelta cadde su una lunga gonna color cammello, che le arrivava alle caviglie e che abbinò a un maglioncino talmente comodo che si sarebbe potuto definire asessuato, dal momento che nascondeva ogni evidenza della sua femminilità.// / Perfino se l'avesse vista senza la sua mantella addosso, $/$ Marco lui avrebbe dovuto essere un uomo davvero disperato per trovare qualcosa di erotico in una simile mancanza di forme. / Nucleo $_{/} / \mathrm{E}$ (CORIS, NARRATRomanzi)

(49') Benchè l'avesse vista senza la sua mantella addosso, lui avrebbe dovuto essere un uomo davvero disperato per trovare qualcosa di erotico in una simile mancanza di forme

40 El valor concesivo solo ha sido reconocido en la bibliografía para la construcción incluso si (Flamenco 1999: 3844), a veces presentada como un nexo consolidado (Santos Río 2003: s.v. incluso si). Sin embargo, el valor concesivo aparece en un repertorio más amplio de estructuras. Una cuestión que merecería más atención y en la que no entraremos aquí es en qué medida el valor escalar del conector favorece la aparición del valor concesivo en contextos contrafactuales, como ponen de manifiesto los usos de otros focalizadores escalares como aun en construcciones como aun cuando.

41 A partir de los datos del corpus podemos afirmar que es mucho más frecuente la construcción perfino/persino + selquando que la correspondiente addirittura + selquando $(0$ y 3 ocurrencias respectivamente), lo que podría constituir otro caso de distribución complementaria.

42 «[... la inferencia pragmática que activan dichas partículas cuando actúan sobre una prótasis condicional conlleva, no sólo la implicación de que hay otra u otras condiciones para las que el consecuente o la apódosis concesiva son verdaderos, sino también la implicación de que el antecedente dado es el elemento más improbable y, por tanto, más sorprendente de todas las alternativas posibles» (Flamenco 1999: 3844). 
De este modo se produce un doble movimiento argumentativo: por una parte, el enunciado en el que se sitúa el focalizador viene a reforzar lo aseverado en enunciados anteriores puntualizando que lo dicho tiene validez en situaciones extremas o no esperadas; por otra parte, la información temporal contenida en el Marco que cae bajo el alcance del focalizador resulta antiorientada respecto de la información que se ofrece en el Núcleo, ya que indica una condición o situación que constituye una objeción a cuanto se afirma en la Unidad de Núcleo. Que el valor antiorientado reside en el propio conector y no únicamente en los estados de hechos representados parece confirmarse cuando empleamos en el mismo contexto otro conector aditivo:

(49”) Alla fine la scelta cadde su una lunga gonna color cammello, che le arrivava alle caviglie e che abbinò a un maglioncino talmente comodo che si sarebbe potuto definire asessuato, dal momento che nascondeva ogni evidenza della sua femminilità. // /Inoltre/Marco se l'avesse vista senza la sua mantella addosso,/Marco lui avrebbe dovuto essere un uomo davvero disperato per trovare qualcosa di erotico in una simile mancanza di forme. /vúcleo $/ / \mathrm{E}$

Este valor concesivo se desarrolla de forma natural en la Unidad de Marco ya que, como es sabido, las concesivas introducen siempre el argumento débil en la construcción argumentativa (Anscombre y Ducrot [1983]1994, Flamenco 1999), lo que favorece su posición antepuesta al Núcleo, que contiene normalmente el argumento fuerte, es decir, el punto central de la argumentación desarrollada en el enunciado o en la secuencia de enunciados. En efecto, siguiendo la hipótesis del dinamismo comunicativo desarrollada por Firbas (1992), las posiciones finales del enunciado -siempre y cuando no haya ningún otro mecanismo prosódico, léxico o sintáctico que interfiera- son las posiciones privilegiadas para vehicular los elementos que el hablante considera informativamente más relevantes (la llamada posición de end focus o de foco informativo, neutro o no marcado).

b) Cuando incluso satura la Unidad de Marco, es decir, cuando aparece sin otro material lingüístico, pierde por completo la función focalizadora ${ }^{43}$ y desempeñan únicamente una función de conexión aditiva (Fuentes 1987: 173, Cuartero Sánchez 2002). En tanto que conector enlaza el enunciado en que se encuentra con los que le preceden indicando que entre ellos existe una relación de coorientación argumentativa, es decir, que ambos apuntan a una misma conclusión. Además, introduce el argumento decisivo o bien uno que tiene mayor relevancia que los anteriores.

${ }^{43}$ La pérdida de la función focalizadora se observa, por ejemplo, en que pueden preceder enunciados negativos: Incluso, ni nos saludó, al contrario de lo que sucede cuando se trata de un focalizador *Incluso Juan no vino. 
(50) Los antiinflamatorios no esteroideos (AINES) y la aspirina son los únicos fármacos recomendados para frenar los síntomas de la artrosis. No obstante, su eficacia en esta patología es bastante limitada. Estos medicamentos alivian el dolor y la inflamación a corto plazo, pero no curan ni mejoran la evolución de la enfermedad. // / Incluso,/Marco su consumo prolongado empeora el estado del paciente./Núcleo // E (CREA, El Mundo, 03/02/2001)

En esta posición incluso tiene mucha mayor orientación discursiva que cuando se encuentra en la Unidad de Núcleo, ya que contribuye de forma decisiva a la construcción textual, indicando que toda la información contenida en el Enunciado se añade a lo anteriormente dicho para apoyarlo, clarificarlo o precisarlo, en un movimiento claro de co-orientación argumentativa.

La adquisición de funciones de conector discursivo en esta posición no es exclusiva del focalizador incluso. Otros focalizadores aditivos del español como también pueden llegar a funcionar como conectores aditivos cuando saturan la Unidad de Marco (Borreguero 2011, De Cesare / Borreguero 2014), aunque el consenso sobre este punto es más controvertido que en el caso de incluso (Cuartero Sánchez 2002) ${ }^{44}$.

44 Véanse estos ejemplos en los que también ocupa la posición inicial de enunciado pero está integrado en Unidades Informativas diferentes. En (a) también aparece integrado en la Unidad de Núcleo y desempeña la función de focalizador siendo su foco «los quioscos de periódicos» (elemento del paradigma "lugares donde se ofrecen productos que familiarizan a los niños con el mundo de las armas", al que también pertenecen «los almacenes» mencionados en el enunciado anterior):

(a) Cuando una cosa se convierte en parte integrante de la vida cotidiana, ya no reparamos en ella. Hoy en día, por ejemplo las armas de juguete se han popularizado tanto que los almacenes de todo tipo venden bazookas, ametralladoras y pistolas para los críos.

"/También los quioscos de periódicos ofrecen sus acostumbradas publicaciones para niños:/Núcleo // pistolas que echan humo y caras feroces que asoman a las cubiertas de las series siempre nuevas de tebeos. (CREA, El diario vasco, 03/06/2001).

En (b) también ocupa la Unidad de Núcleo pero su posición preverbal posibilita que su alcance abarque todo el enunciado y no solo un constituyente, lo que le permite al intérprete construir inferencialmente una relación de adición entre este enunciado y un enunciado anterior (en cursiva en el texto) donde se menciona que el primer premio en el concurso de escaparates lo ha ganado la Mercería Leintz por primera vez:

(b) El jurado que debía deliberar sobre los escaparates que entraban a concurso esta edición decidió que el primer premio consistente en 35.000 pesetas y placa, recayera en la Mercería Leintz, cuya responsable es Rosi Martínez de la Hidalga. Se da la circunstancia de que es la primera vez que gana desde que en 1988 se comenzara a hacer en Aretxabaleta el concurso de escaparates. El jurado ha tenido en cuenta la sencillez y mensaje del escaparate que se ha llevado el primer galardón.

El segundo premio, de 25.000 pesetas, se lo ha llevado la Peluquería Felix (Katxo). // /También es la primera vez que recibe el premio si bien es cierto que concursa por segunda vez./Núcleo // (CREA, El diario vasco, 06/01/2001).

En (c) también aparece saturando la Unidad de Marco (separada gráficamente del resto del enunciado por una coma) y no realiza ya su habitual función de focalización sino que se comporta como un conector aditivo equivalente a además. Véase la correspondencia con igualmente que 
En el caso del italiano, sin embargo, el único focalizador escalar aditivo que puede adoptar la función de conector aditivo es addirittura:

(51) Ferrari è anche l'unica azienda che partecipa al campionato mondiale sin dal primo anno, il 1950. Per la casa di Maranello, le competizioni sono sempre state un fondamentale terreno di prova per la produzione delle Gran Turismo da vendere ai clienti. // Addirittura, /Marco sino agli anni '70,/Marco sono stati spesso gli stessi clienti della Ferrari a fare da collaudatori per la casa del cavallino rampante./Núcleo $/ /{ }_{\mathrm{E}}$ (CORIS, STAMPASupplementi)

$\left(51^{\prime}\right)^{*}$ Perfino,/Marco sino agli anni '70,/Marco sono stati spesso gli stessi clienti della Ferrari a fare da collaudatori per la casa del cavallino rampante

Sin embargo, constituyen buenos equivalentes de incluso cuando satura la Unidad de Marco ciertos conectores aditivos escalares como inoltre (52) e in più (53), que también se corresponden con con el conector correspondiente en español además (Cuartero Sánchez 2002, Portolés 2012, 2013) ${ }^{45}$. No obstante, estos conectores aditivos no son intrínsecamente escalares, lo que supone que su valor escalar depende fundamentalmente del contexto en que se hallen (un buen ejemplo de conector aditivo escalar intrínseco es el español encima).

(52) La tradizione Italiana, ad esempio, usa associare al Biancospino altre droghe sedative come la Camomilla (Wichtl, 1993, pag. 161-163). A tal proposito esistono anche riscontri sperimentali: la somministrazione orale di estratti di Crataegus levigata nel topo ha prodotto, in una batteria di quattro

funciona también como conector aditivo en el enunciado E2 y contribuye a establecer un paralelismo entre la estructura de ambos enunciados:

(c) Otra línea nueva está dirigida a compensar los gastos derivados de la destrucción de los restos animales que mueren en la explotación, aplicable en el marco del Plan sobre la Encefalopatía Espongiforme Bovina.// /También,/Marco en el próximo año se ampliará la garantía de daños por falta de nascencia a los cultivos de secano que no disponen de ella en el seguro de rendimientos de cultivos herbáceos extensivos./Núcleo // $\mathrm{E} 1 /$ Igualmente,/Marco en el seguro de frutales, se mejorarán las condiciones de aseguramiento para fomentar la fidelización de su contratación. // E2 (CREA, Diario de Navarra, 09/01/2001).

${ }_{5}$ Más problemático es el caso de per di più que en muchos diccionarios aparece como sinónimo de inoltre y de in più y que también puede traducirse por además (pero no por incluso) en muchos contextos. Per di più, a nuestro juicio, es un conector aditivo que introduce un argumento no necesariamente central para la conclusión a la que se quiere llegar, sino más bien marginal o añadido o superfluo y que por tanto no pertenece necesariamente a la misma escala que los argumentos presentados anteriormente. Así, en el siguiente ejemplo, la tesis fundamental (la escasa cortesanía del protagonista) está apoyada por la información relativa a los años transcurridos como esclavo en una plantación, pero reforzada por un argumento adicional (la complejidad de los bailes de moda) que, sin embargo, no es central para la argumentación. «Le sue maniere, prima raffinate, risultavano brusche a confronto con la disinvoltura dei cubani. Gli anni trascorsi nella piantagione lo avevano indurito dentro e fuori e ora, quando più ne aveva bisogno, era privo di quelle arti cortigiane che tanto gli erano naturali in gioventù. Per di più, i balli di moda erano un rapido groviglio di piroette, riverenze, giri e saltelli che non era in grado di imitare». (CORIS, MON2008_10). 
distinti modelli sperimentali, una netta, anche se moderata, azione depressiva sul sistema nervoso centrale. // / Inoltre, $/$ Marco una sperimentazione clinica multicentrica, controllata con placebo ha dimostrato che il Biancospino somministrato insieme a Valeriana, Passiflora, Ballota, Cola e Paullinia (Euphytose $(\mathrm{r}))$ - risulta efficace in pazienti con disturbi comportamentali con note ansiose. // (CORIS, MISCVolumi)

(53) Il rialzo delle tariffe, appunto, e il taglio dei trasferimenti alle aziende di trasporto, oltre all'obbligo di una forzato risparmio sui costi. Tradotto: la Regione stringerà i cordoni della borsa. Più penalizzati saranno i servizi su gomma che subiranno un abbattimento dei contributi tra il 7 e 12 per cento. Meglio andrà per le aziende ferroviarie: il taglio è calcolato tra lo zero e il cinque per cento. // /In più,/Marco a tutte le imprese di trasporto pubblico sarà imposta una dieta dimagrante del tre per cento, alla voce «razionalizzazione». In totale, le aziende si troveranno con una disponibilità di cassa diminuita del dieci per cento. (CORIS, MON2008_10)

\section{FOCALIZADORES ADITIVOS ESCALARES ENLA UNIDAD DEAPÉNDICE}

Cuando incluso aparece en la Unidad de Apéndice su función varía considerablemente dependiendo de si va acompañado por otros elementos lingüísticos o no, como ya ocurría en la Unidad de Marco.

En el primer caso, añade una información que precisa o complementa la información del Núcleo o del Marco al que acompaña, pero indicando que esa información resulta de algún modo la menos esperada dentro de un conjunto de alternativas explícitas o implícitas (54). Esta información se sitúa en un plano secundario respecto de la información principal (en (54), por ejemplo, que la terapia se puede aplicar con garantías), de modo que el elemento focalizado no constituye el foco del enunciado como ocurría en la Unidad de Núcleo. En estos casos, por tanto, es necesario hablar de dos focos diferentes: el foco del enunciado (con garantías en (54)) y el foco del focalizador (los pacientes que no cumplen requisitos estrictos de selección en (54)).

(54) Es posible que las diferencias entre los resultados alcanzados por los pacientes más motivados y los menos motivados residan en la frecuencia de los controles de glucosa sanguínea, ya que en los estudios en los que ha participado el primer grupo de pacientes estos controles son mucho más frecuentes. // / No obstante,/Marco cuando se proporciona información detallada,/Marco la terapia intensiva (II.MM. o ISCI) se puede aplicar con garantías,/Núcleo incluso a pacientes que no cumplen requisitos estrictos de selección. / $^{\text {péndice }} / / \mathrm{E}_{\mathrm{E}}$ (CREA, Informe de evaluación de tecnologias sanitarias, 2000)

Según la posición del Apéndice del Núcleo se puede distinguir el Apéndice Extensivo, que se sitúa como última Unidad Informativa del Enunciado y 
permite un desarrollo más extenso de la información secundaria, y el Apéndice Intensivo, intercalado en el Núcleo y más breve, como (55):

(55) Precisamente el título de esta exposición, Recorridos, recoge esta idea, ya que se trata de una búsqueda de signos emblemáticos que Etchegaray recrea en colores vivos, / incluso opuestos, /Apéndice en obras que mezclan los géneros y las esculturas. (CREA, El Diario Vasco, 13/03/2001)

También es posible, pero menos frecuente, que el Apéndice informativo siga a la Unidad de Marco, como en (56):

(56) La noticia de la retirada del candidato oficial danés cayó como un auténtico jarro de agua fría entre los políticos daneses y aún más entre los medios informativos y el resto de la población. Uffe Ellemann-Jensen es un personaje muy popular y querido en Dinamarca.

Aunque es el líder de la oposición, el Gobierno de coalición que preside el socialdemócrata Poul Nyrup-Rasmussen ha tenido un excepcional interés en la candidatura oficial de Ellemann-Jensen, que casi tenía el signo de una cruzada. // /Para los daneses,/Marco incluso para el Gobierno,/Apéndice era el honor nacional lo que estaba en juego y, por primera vez en la historia de la política danesa, se enterraron las hachas de diaria guerra política, unificando fuerzas para conseguir que Uffe Ellemann-Jensen ocupara el sillón de la OTAN en Bruselas. // (CREA, La Vanguardia, 02/12/1995).

En todos estos casos, incluso funciona como un focalizador y, por tanto, sus equivalentes italianos son los que ya hemos visto en la Unidad de Núcleo: perfino/persino y addirittura. Sin embargo, a diferencia de lo que ocurría en esta última, el foco (junto con el focalizador) satura la Unidad de Apéndice en todos los casos:

(57) La forma più diffusa di maltrattamenti contro le donne e le bambine in tutto il mondo è la violenza esercitata su di loro dai loro stessi partner:// un'altissima percentuale di donne sono vittime ogni anno,/ Núcleo- $^{\text {perfino }}$ in paesi sviluppati come l'Australia, il Canada, Israele o gli Stati Uniti,/Apendice dei loro stessi mariti e fidanzati, attuali o ex./-Núcleo // E (CORIS, MON2005_07)

Cuando incluso aparece en un Apéndice extensivo -independientemente de si el Apéndice aparece gráficamente separado por comas de la Unidad de Núcleopuede asumir una función reformulativa (López Serena / Loureda 2013), de tal modo que el segmento discursivo en que se encuentra se interpreta como una información que el locutor añade -tras un momento de pausa o vacilación-para aclarar o especificar lo dicho anteriormente en el Núcleo. Este efecto se acentúa especialmente en los casos en que el focalizador aparece pospuesto al elemento focalizado. Esta reformulación no tiene en ningún caso valor sustitutivo, es decir, 
la información en Apéndice no puede considerarse vinculante o decisiva, porque de ese modo anularía o minimizaría la relevancia comunicativa de lo dicho en el Núcleo ${ }^{46}$. Su finalidad comunicativa es la de aclarar el contenido del Núcleo por medio de una información que se añade en un segundo momento, que permanece subordinada a dicho Núcleo y que, por lo general, hace referencia a un grado superior o inferior respecto de una entidad o cualidad cuantificable mencionada en el Núcleo.

En (58) tras haber calificado de «desequilibrado» al padre Surin, el autor del texto añade en Apéndice un calificativo que en la escala semántica ocupa un lugar más elevado por indicar un grado mayor de la cualidad referida (desequilibradopsicótico), pero que no es incorporado a la información nuclear aseverada por el autor, sino que constituye una observación marginal, como si el autor nos dijera "se podría decir que era un psicótico pero dejémoslo estar":

(58) Ha habido grandes personajes santos tenidos por el colmo de la espiritualidad, como santa Gema Galgani, perturbada mental para su confesor monseñor Volpi, y que otros testigos solventes dudaron de sus pretendidos dones extraordinarios, que eran trastornos psíquicos; // /y el famoso jesuita padre Surin, $/^{\text {Marco }}$ hoy se sabe que era un desequilibrado,/Núcleo un psicótico incluso,/Apéndice1 de acuerdo con los estudios de su vida y obras hechos por el profesor de psiquiatría, el católico De Greeff./Apéndice2 // (CREA, E. Miret Magdalena, ¿Qué nos falta para ser felices?, Barcelona, 2002)

En este caso, además de addirittura (59) y perfino/persino (60), sería posible emplear como equivalente italiano anzi, como se ve en (61), si bien aquí el valor reformulador es más transparente:

(59) Mai tanti fiumi di parole sono stati sprecati per una "pellicola" così banale,/ inutile, addirittura, /Apéndice a mio parere dannosa ...(CORIS, EPHEMOpuscoli) (60) [...] e la sua voce profonda, una voce sonora che arrivava da qualche recesso del diaframma, la trovavo terribilmente sexy e fu sempre così, e poi la sua risata - che era molto più acuta, a volte quasi stridula, una risata che sembrava venir fuori dalla gola,/ dalla testa perfino, ${ }^{\text {Apéndice }}$ e ogni volta che qualcosa le faceva il solletico alla punta del gomito - adesso sto parlando di dopo, non di quella sera - la prendeva una ridarella folle ... (CORIS, NARRATRomanzi)

(61) Tutti quelli che hanno il mal di testa pigliano l'aspirina e gli passa. $\mathrm{Ma}$ è possibile che si possa rendere la vita della gente più igienica e curare il tracoma senza che dalla cura del tracoma si arrivi presto alla fabbrichetta dell'industriale di Hong Kong? Questa è una domanda giusta, non ti pare? FOLCO: Sì. TIZIANO: Ed è un problema che un visitatore come me sente forte, perché presto, / immediatamente anzi,/Apéndice ti rendi conto che tu stesso

46 Distinta es la interpretación de Portolés (2009: n. 15) que, siguiendo a van der Auwera, habla en estos casos de escalas sustitutivas (y no aditivas), en las que el elemento focalizado sustituye a una alternativa con menor valor informativo. 
hai in parte messo in moto quel processo della modernizzazione. (CORIS, MON2005_07)

Cuando incluso satura la Unidad de Apéndice, es necesario tener en cuenta, para determinar su función discursiva, si se trata de una Unidad de Apéndice que depende de la Unidad de Marco o de la Unidad de Núcleo. En el primer caso, incluso tiene un alcance claramente discursivo, muy similar al que adquiere cuando satura la Unidad de Marco e indica las relaciones de conexión aditiva existentes entre dos o más Enunciados pero desde una perspectiva menos prominente:

(62) Los responsables del estudio establecen que, aunque la información a los menores es siempre interesante y formativa, son los adultos los responsables de lo que ponen en la mesa diaria de sus hijos. // Por ello,/Marco incluso,/Apéndice se estudia la posibilidad de dirigir próximos trabajos a este colectivo./Núcleo // (CREA, El Norte de Castilla, 14/02/2001)

En esta posición, solo es posible el focalizador italiano addirittura:

(63) la crisi dei 50 anni non esiste. È un mito.// Forse,/Marco addirittura,/Apéndice "una congiura tra venditori di auto usate e sociologi disoccupati":/Núcleo // $\mathrm{E}$ le due categorie che della crisi dei 50 anni avrebbero fatto una lucrosa industria. (CORIS, STAMPAPeriodici)

En cuanto al Apéndice de Núcleo, cuando aparece inserto en el Núcleo, mantiene su función de focalizador, pero la posición parentética le otorga un carácter menos enfático respecto de la opción en la que aparecía integrado en el Núcleo, formando parte de este:

(64) Durante muchos siglos, los atisbos y genialidades de los griegos clásicos dieron lugar a una rica y variada tradición cultural. En ella se mezclan saberes espaciales, esfuerzos intelectuales, exploraciones y descubrimientos, curiosidad, necesidades prácticas, ideas y creencias, prejuicios de distinto orden, que constituyen el magma cultural en el que la geografía moderna ha tendido a reconocer una tradición propia. // /Para muchos autores,/Marco geógrafos y no geógrafos, $/{ }^{\text {Apéndice }}$ se trata, $/^{\text {Núcleo- }}$ incluso, $/$ Apéndice de la historia de la geografía./-Núcleo // De ahí el interés y la atención prestada a esta primera parte, desde la doble perspectiva del valor intrínseco de esta tradición de saberes y prácticas, y de la necesidad de establecer las diferencias esenciales que separan esa tradición del proyecto moderno de geografía. (CREA, Ortega, J., Los horizontes de la geografía, Alicante, 2000). 
Véase, por ejemplo, la diferencia entre (65) y $\left(65^{\prime}\right)$ :

(65) En silencio, pausadamente, había dedicado los últimos años no solo al estudio de los ovnis, sino a adentrarse en el farragoso mundo de los supuestos contactos directos con los presuntos extraterrestres. Nada dado a la publicidad de sus convicciones, compartía vitalmente las veinticuatro horas del día entre sus muchas actividades como religioso y esa afición que seguía abarcando una importante porción de su alma. // /Él,/Núcleo- incluso,/Apéndice estaba implicado en primera persona en uno de esos casos en los que los ovnis aparecían previo mensaje anunciador en la fecha y el lugar indicado./-Núcleo // (CREA, Jiménez, I., Enigmas sin resolver II, Madrid, 2000).

(65’) En silencio, pausadamente, había dedicado los últimos años no solo al estudio de los ovnis, sino a adentrarse en el farragoso mundo de los supuestos contactos directos con los presuntos extraterrestres. Nada dado a la publicidad de sus convicciones, compartía vitalmente las veinticuatro horas del día entre sus muchas actividades como religioso y esa afición que seguía abarcando una importante porción de su alma. // /ncluso él estaba implicado en primera persona en uno de esos casos en los que los ovnis aparecían previo mensaje anunciador en la fecha y el lugar indicado./Núcleo //.

La posición de incluso en Apéndice y pospuesto al sujeto hace pasar a segundo plano la focalización respecto de su posición integrada en el Núcleo. En estos casos, el italiano muestra una clara preferencia por addirittura (66), aunque también se encuentran casos con perfino/persino, con frecuencia intercalados entre el Núcleo y un segundo Apéndice (67) o un Inciso (68).

(66) È anzitutto la netta sensazione o, meglio, l'inquietante certezza, di non essere riconfermati perché il proprio partito di appartenenza, di solito piccolo e senza un gran peso politico, ha perso, o sta perdendo, consensi e rischia,/ addirittura,/Apéndice l'estinzione. (CORIS, STAMPAPeriodici)

(67) Il caso del pazzo che disegna sagome, lo chiamano. Lo fa di notte, notte fonda, con il gessetto bianco. Sui marciapiedi, sulle strade, a pochi metri dai portoni delle case. Sagome come quelle che poliziotti o carabinieri tracciano sull'asfalto dopo un incidente mortale o un omicidio. Uomini con le braccia allargate e una gamba piegata; donne con i capelli sparsi sul selciato, la borsa abbandonata poco lontano, sagoma anch'essa; // /bambini,/Núcleo, perfino, / ${ }^{\text {Apéndice }}$ corti perimetri disegnati in prossimità delle scuole./Apéndice // (CORIS, NARRATRacconti)

(68) // /Avevo avuto fortuna,/Núcleo perfino,/Apéndice // ${ }_{E 1}$ come mi riferì l'ingegnere che coadiuvava l'intera baracca: // 2 il mio predecessore si era beccato ben 2000 millirem nel giro di soli undici mesi. (CORIS, NARRATRacconti) 


\section{CONCLUSIONES}

Como hemos tratado de mostrar por medio de este análisis, la articulación del nivel informativo textual en Unidades Comunicativas (Enunciados) y Unidades Informativas (Marco, Núcleo, Apéndice) que establece el modelo de Basilea permite identificar los distintos valores discursivos de un elemento con una minuciosidad que, a nuestro juicio, dista bastante de la habitual en los estudios con un corte puramente sintáctico o puramente pragmático. El acierto del modelo estriba precisamente, como se ha dicho, en considerar la interacción entre el valor semántico codificado del elemento o la estructura estudiada y los valores contextuales que se desarrollan a partir de su inserción en un entramado textual.

En el caso de los adverbios analizados en este trabajo (incluso, addirittura, persino/perfino) el valor aditivo escalar constituye su valor nuclear y, en tanto que partículas discursivas, codifican una instrucción relativa al procesamiento del segmento discursivo en que aparecen que podría parafrasearse de la siguiente manera: el elemento sobre el que inciden se añade a otros, explícitos o implícitos en el cotexto inmediato, que pertenecen a un mismo paradigma. Además, el elemento sobre el que inciden es presentado como un elemento informativamente más relevante porque, dentro de la escala pragmática en la que se ordenan los miembros de ese paradigma, transmite una información que resulta menos predecible para el destinatario o, al menos, el locutor considera que el destinatario no puede deducirla de la información precedente, es decir, que no forma parte en ningún caso de la información de trasfondo o presupuesta.

A partir de estos valores semánticos nucleares, se desarrollan distintas funciones discursivas -focalizador, conector, reformulador- que emergen cuando estos elementos aparecen en una determinada Unidad Informativa y simultáneamente (o no) con otro material lingüístico en la misma Unidad. En el análisis contrastivo a cada función discursiva le corresponde un equivalente diverso, como hemos tratado de poner de manifiesto en este trabajo. Así, la presencia de otros elementos léxicos adyacentes, que quedan bajo su alcance inmediato, favorece su interpretación como adverbios focalizadores, tal y como ha sido señalado en la bibliografía (Ricca 1999, Andorno 2000, Schwenter 2000, Portolés 2007, 2008, 2009, Ferrari et al. 2011). En estos contextos lo que prima es poner de relieve la importancia informativa del constituyente focalizado y establecer el contraste entre dicho constituyente y los que componen la alternativa.

Esta función focalizadora central aparece especialmente en las ocurrencias de incluso, addirittura y perfino/persino en la Unidad Informativa de Núcleo, donde el segmento discursivo focalizado puede llegar a constituir el Foco del 
Núcleo si ocupa la posición final dentro de esta Unidad ${ }^{47}$. Por otra parte, en los casos en que incluso satura la Unidad de Núcleo, el destinatario se ve obligado a recuperar inferencialmente, a partir del contexto, el elemento focalizado. Pero el focalizador perfinolpersino no aparece en este tipo de contextos y, por tanto, no puede funcionar como equivalente de incluso.

Cuando se amplía el número de los constituyentes que quedan bajo el alcance del focalizador se diluye en cierto modo la función focalizadora y se resalta el valor aditivo, lo que puede llevar al destinatario a establecer una conexión inferencial de tipo aditivo entre el enunciado en el que aparece incluso y los enunciados precedentes. También en este caso los focalizadores aditivos escalares del italiano se comportan en modo diverso, ya que perfino / persino-seguramente por su origen preposicional- necesariamente aparece en posición adyacente al elemento focalizado y no puede extender su alcance a todo un enunciado, mientras addirittura se comporta a este respecto del mismo modo que incluso.

El valor aditivo cobra especial relevancia cuando incluso aparece en la Unidad de Marco acompañado de otro material lingüístico. La Unidad de Marco se proyecta informativamente tanto a su derecha como a su izquierda. Por una parte, la información contenida en dicha unidad indica las circunstancias espacio-temporales y modales de la acción que aparecen focalizadas por incluso, circunstancias que pueden mantener su vigencia no solo para el enunciado en el que aparecen sino también para los enunciados siguientes (proyección a la derecha). Por otra parte, en su proyección hacia la izquierda, la aparición de incluso en la Unidad de Marco refuerza la conexión entre el enunciado en que aparece y el precedente. Esto es así especialmente cuando la información se presenta bajo la forma de una subordinada adverbial de cierta extensión, de modo que resulta difícil identificar con precisión el elemento focalizado. En estos casos la función focalizadora parece diluirse a favor de la función de conexión lógicoargumentativa. Si, además, la subordinada es de tipo condicional o temporal, incluso desarrolla un valor de tipo concesivo-condicional, de tal modo que crea un movimiento argumentativo anti-orientado dentro del enunciado. La información transmitida en la subordinada (en la prótasis) expresa cierta reserva o condición improbable, que, sin embargo, no es obstáculo para que tenga lugar el hecho que constituye la información nuclear del Enunciado. Obsérvese que el mismo efecto de sentido se produce con addirittura y con perfino y cabe pensar que a partir de esta combinación se ha gramaticalizado el conector concesivo anche se.

La función propiamente conectiva se desarrolla de modo exclusivo cuando incluso aparece saturando la Unidad de Marco. En estos casos, parece claro que no hay ningún elemento focalizado y que el papel que desempeña incluso es el de

${ }^{47}$ En caso contrario, la Unidad de Núcleo puede tener un Foco propio en posición final que no coincide con el foco del adverbio focalizador (De Cesare 2010). 
crear un vínculo lógico-argumentativo de naturaleza aditiva entre el enunciado al que precede y el enunciado o grupo de enunciados anteriores. En esta posición ya no puede hablarse de adverbio focalizador sino de conector aditivo escalar y sus usos coinciden con los de otros conectores como es más o además (en los casos en que admite una interpretación escalar). Con estos usos, los equivalentes italianos más próximos son, además de addirittura, inoltre e in più.

Por último, en la Unidad de Apéndice tenemos que distinguir igualmente dos funciones de incluso determinadas por su combinación o no con otro material lingǘstico dentro de la misma unidad. Si incluso aparece en un Apéndice inserto en el Núcleo, tanto acompañado de otros elementos léxicos como solo, su función es exclusivamente focalizadora y, por tanto, puede sustituirse por cualquiera de los dos focalizadores escalares italianos. Se trata, sin embargo, de una focalización que se mantiene en un segundo plano respecto de la información nuclear, como corresponde al estatus de esta Unidad Informativa. En algunas ocurrencias, especialmente cuando aparece en una Unidad de Apéndice extensiva y además pospuesto al elemento focalizado, incluso adquiere un valor reformulativo, pero se trata en cualquier caso de una reformulación que no tiene carácter sustitutivo respecto de la información focal de la Unidad de Núcleo y que simplemente matiza, aclara o precisa dicha información, por lo que solo en determinados contextos es equivalente a un verdadero reformulador como anzi.

Cuando incluso aparece saturando una Unidad de Apéndice inserta o posterior a una Unidad de Marco, adquiere función de conector aditivo, pero a diferencia de sus ocurrencias en esta última Unidad, su aparición en una Unidad de Apéndice vinculada a un Marco tiene un alcance mucho más limitado, de modo que no puede establecer una conexión entre el enunciado y un bloque de enunciados precedentes, sino entre dos Unidades Informativas o, a lo sumo, entre el Enunciado que lo contiene y el inmediatamente precedente. Como hemos visto, de los adverbios escalares italianos, solo addirittura asume en esta posición una función de conector aditivo.

Sin embargo, si la Unidad de Apéndice saturada por incluso está vinculada a la Unidad de Núcleo, prevalece el valor focalizador, aunque se trata en cualquier caso de una focalización mitigada, precisamente por la aparición del adverbio en una posición enunciativa reservada para la información secundaria.

Como síntesis del estudio que hemos llevado a cabo en este trabajo presentamos las principales funciones discursivas de incluso y los equivalentes italianos que le corresponden en cada caso en la siguiente tabla (hemos añadido también el focalizador escalar no intrínseco anche): 


\begin{tabular}{|c|c|c|c|}
\hline Unidad Informativa & \multirow[t]{2}{*}{ Función discursiva } & \multirow[t]{2}{*}{ Ocurrencias de incluso } & \multirow[t]{2}{*}{ Equivalente italiano } \\
\hline Unidad de Núcleo & & & \\
\hline & $\begin{array}{l}\text { Focalizador con } \\
\text { foco explícito }\end{array}$ & Ha venido incluso Juan & $\begin{array}{l}\text { Perfino/persino } \\
\text { Addirittura } \\
\text { Anche }\end{array}$ \\
\hline & $\begin{array}{l}\text { Focalizador con } \\
\text { foco inferido }\end{array}$ & $\begin{array}{l}\text { He hecho la cena y } \\
\text { he lavado los platos. } \\
\text { Incluso! }\end{array}$ & $\begin{array}{l}\text { Addirittura! } \\
\text { Anche! }\end{array}$ \\
\hline & $\begin{array}{l}\text { Focalizador con } \\
\text { alcance sobre todo } \\
\text { el enunciado }\end{array}$ & $\begin{array}{l}\text { Incluso ba dicho que me } \\
\text { va a regalar un coche }\end{array}$ & Addirittura \\
\hline \multicolumn{4}{|l|}{ Unidad de Marco } \\
\hline & $\begin{array}{l}\text { Focalizador con } \\
\text { foco explícito }\end{array}$ & $\begin{array}{l}\text { Incluso en invierno, se } \\
\text { baña en el mar todas las } \\
\text { mañanas }\end{array}$ & $\begin{array}{l}\text { Perfino/persino } \\
\text { Addirittura } \\
\text { Anche }\end{array}$ \\
\hline & $\begin{array}{l}\text { Focalizador con } \\
\text { alcance sobre una } \\
\text { estructura temporal } \\
\text { o condicional y } \\
\text { valor concesivo }\end{array}$ & $\begin{array}{l}\text { Incluso cuando no tengo } \\
\text { que ir a trabajar me } \\
\text { levanto a las seis }\end{array}$ & $\begin{array}{l}\text { Perfinolpersino } \\
\text { Anche }\end{array}$ \\
\hline & Conector aditivo & $\begin{array}{l}\text { Incluso, } \\
\text { manifestantes algunos } \\
\text { encontrados inconscientes } \\
\text { sobre la calzada }\end{array}$ & $\begin{array}{l}\text { Addirittura } \\
\text { Inoltre, In più }\end{array}$ \\
\hline \multicolumn{4}{|l|}{ Unidad de Apéndice } \\
\hline & $\begin{array}{l}\text { Focalizador con } \\
\text { foco explícito }\end{array}$ & $\begin{array}{l}\text { Todos, incluso los } \\
\text { abuelos, se animaron a } \\
\text { bailar }\end{array}$ & $\begin{array}{l}\text { Perfino/persino } \\
\text { Addirittura } \\
\text { Anche }\end{array}$ \\
\hline & $\begin{array}{l}\text { Reformulador } \\
\text { (pospuesto al foco) }\end{array}$ & $\begin{array}{l}\text { La comida estaba fría, } \\
\text { helada incluso. }\end{array}$ & $\begin{array}{l}\text { Perfino/persino } \\
\text { Addirittura } \\
\text { Anzi (ं?) }\end{array}$ \\
\hline & $\begin{array}{lr}\text { Conector } & \text { aditivo } \\
\text { (pospuesto } & \text { al } \\
\text { Marco) } & \\
\end{array}$ & $\begin{array}{l}\text { Desde entonces, incluso, } \\
\text { me envia una caja } \\
\text { de bombones por mi } \\
\text { cumpleaños. }\end{array}$ & $\begin{array}{l}\text { Addirittura } \\
\text { Inoltre, In più }\end{array}$ \\
\hline & $\begin{array}{lr}\text { Focalizador } & \text { con } \\
\text { foco implícito } \\
\text { (pospuesto r al } \\
\text { Núcleo) }\end{array}$ & $\begin{array}{l}\text { Trabajamos mucho. Los } \\
\text { jefes, incluso, van a la } \\
\text { oficina los sábados por la } \\
\text { tarde. }\end{array}$ & $\begin{array}{l}\text { Addirittura } \\
\text { Perfino/persino }\end{array}$ \\
\hline
\end{tabular}


Este análisis pone de manifiesto, a nuestro juicio, cómo las distintas funciones discursivas de estos adverbios no dependen exclusivamente de su alcance o de su realización prosódica, paréntetica o integrada, sino que están determinadas de modo decisivo por su aparición en las distintas Unidades Informativas del Enunciado. Sin que sea posible establecer una correspondencia rígida y automática que permitiera asignar un valor indiscutible para cada Unidad Informativa, sí parece claro que las Unidades de Núcleo y Apéndice de Núcleo favorecen la función de focalizador, mientras que las Unidades de Marco y Apéndice de Marco se especializan en la de conector aditivo. A estas dos grandes funciones, se suman en ocasiones otras que tienen sin duda carácter secundario respecto a las primeras: así la función de reformulación en los Apéndices de Núcleo de carácter extensivo o la función de conector concesivo en la Unidad de Marco.

La utilidad del Modelo de Basilea para el estudio contrastivo estriba en que permite afinar el análisis hasta el extremo de poder desentrañar las diferencias entre dos focalizadores escalares, como persino/perfino y addirittura, que con frecuencia se consideran idénticos $y$, por añadidura, equivalentes válidos para incluso en cualquier contexto. Como hemos visto, el único que realmente puede desempeñar todas las funciones de incluso en cualquier posición enunciativa y con cualquier función discursiva es addirittura (excepto cuando precede a una subordinada temporal o condicional y tiene valor concesivo), mientras que perfino/persino tiene un uso mucho más restringido, limitado, por lo general, a la focalización directa de un elemento explícito independientemente de la Unidad Informativa en que se encuentre. De hecho, no puede aparecer en la Unidad de Núcleo si su foco no está claramente restringido a un constituyente sintagmático y el alcance se extiende a todo el enunciado. Tampoco puede saturar una Unidad de Núcleo (con el foco implícito) ni de Marco o de Apéndice de Marco, posiciones estas últimas que propician el desarrollo de una función conectora bloqueada para persino/perfino. Más notable es la presencia, inesperada, de persino/perfino saturando la Unidad de Apéndice del Núcleo, lo que nos refuerza en la hipótesis de que, a pesar del carácter parentético de esta unidad, el adverbio ejerce una función claramente focalizadora sobre uno de los constituyentes presentes en el Núcleo.

El estatus informativo de cada una de estas Unidades constituye, por tanto, un factor decisivo para la asignación de funciones discursivas en los distintos contextos de aparición de las partículas discursivas polifuncionales o, al menos, para poder establecer una jerarquía mínima entre la función primaria o principal en una determinada ocurrencia y otras funciones secundarias. La interacción entre el valor semántico nuclear de cada partícula y su integración en la estructura informativa del Enunciado posee mayor valor heurístico que otras aproximaciones teóricas basadas únicamente en factores sintácticos como el alcance del focalizador, la naturaleza del foco o la posición de la partícula 
focal respecto del foco. Eso explica también que, por lo general, la mayoría de los estudios sobre estos adverbios hayan prestado especial atención a su función focalizadora sin tener en cuenta otras funciones discursivas o no hayan reparado en la importancia de la interacción entre la partícula y la estructura informativa como base para la explicación de la polifuncionalidad paradigmática tan características de estos elementos.

\section{BIBLIOGRAFÍA}

Aijmer, K. - Simon-Vandenbergen, A. - M., "Pragmatic markers", en J. Zienkowski et al (eds.), Discursive Pragmatics, Amsterdam, John Benjamins, 2011, págs. 223-247.

Andorno, C., "Avverbi focalizzanti in italiano. Parametri per un'analisi", Studi Italiani di Linguistica Teorica e Applicata, 28/1 (1999), págs. 43-83.

Andorno, C., Focalizzatori fra connessione e messa a fuoco. Il punto di vista delle varietà di apprendimento, Milano, Franco Angeli, 2000.

Anscombre, J. C. - Ducrot, O., L'argumentation dans la langue, Bruxelles, Mardaga, 1983. [Trad. esp. de Marta Tordesilla, La argumentación en la lengua, Madrid, Gredos, 1994].

Arquès, R. - Padoan, A. (coords.), Il Grande dizionario di spagnolo. Dizionario spagnoloitaliano, italiano-spagnolo, Bologna, Zanichelli, 2012.

Bermejo Calleja, F., "Aproximación contrastiva al estudio del adverbio en italiano y en español", en F. San Vicente (ed.), Partículas. Particelle. Estudios de lingüistica contrastiva español italiano, Bologna, Clueb, 2007, págs. 179-195.

Bermejo Calleja, F., "El adverbio", en F. San Vicente (dir.), GREIT. Gramática de referencia de español para italófonos, Bologna, CLUEB / Salamanca, Universidad de Salamanca, 2013, vol. I, págs. 405-437.

Blakemore, D., Semantic constraints on relevance, Oxford, Blackwell, 1987.

Blanche-Benveniste, C. et al., Le français parlé. Etudes grammaticales, Paris, Editions du CNRS, 1990.

Briz, A., grupo Val. Es.Co., "Un sistema de unidades para el estudio del lenguaje coloquial", Oralia, 6 (2003), págs. 7-61.

Briz, A. - Pons, S. - Portolés, J. (dirs.), Diccionario de partículas del español. <www. dpde.es>

Borreguero Zuloaga, M., "Focalizzatori a confronto: anche vs también", Studi italiani di linguistica teorica e applicata XL:3 (2011), págs. 441-468.

Borreguero Zuloaga, M., "Left-periphery in discourse: Frame Units and discourse markers", en A. Dufter - A. Octavio de Toledo (eds.), Left-peripheries in Spanish. Diachronic, typological and variationalist approaches, Amsterdam, John Benjamins, 2014, págs. 345-382.

Cignetti, L., L'Inciso. Natura linguistica e funzioni testuali, Alessandria, Ed. dell'Orso, 2011.

Cortés, L. - Cамacho, M. M., Unidades de segmentación y análisis del discurso, Madrid, Arco Libros, 2002.

Cresti, E., Corpus di italiano parlato, Firenze, Accademia della Crusca, 2000, 2 vols. 
Cuartero Sánchez, J. M., Conectores y conexión aditiva. Los signos incluso, también y además en el español actual, Madrid, Gredos, 2002.

De Cesare, A. M., Intensification, modalisation et focalisation. Les différentes effets des adverbes proprio, davvero et veramente, Bern, Lang, 2002a.

De Cesare, A. M., "Gli usi e le funzioni dell'avverbio proprio", Italica, 79/4 (2002b), págs. 453-465.

De Cesare, A. M., "Y a-t-il encoré quelque chose à ajouter sur l'italien anche? Une réponse basée sur le CORIS/CODIS", Rivista di linguistica/Italian Journal of Linguistics, 16/1 (2004a), págs. 3-34.

De Cesare, A. M., "L'avverbio anche e il rilievo informativo del testo", en A. Ferrari (ed.), La lingua nel testo, il testo nella lingua, Torino, Istituto dell'Atlante Linguistico Italiano, 2004b, págs. 191-218.

De Cesare, A. M., "Soprattutto tra avverbio focalizzante e congiunzione testuale", en E. Corino et al. (eds.), Proceedings XII EURALEX International Congress. Congresso internazionale di lessicografia (Torino, 6-9.9.2006), Alessandria, Edizioni dell'Orso, 2006, II, págs. 1129-1135.

De Cesare, A. M., "A corpus-based approach to the concept of synonymy: A case study based on the Italian anche and pure", en J. Cihlar et al. (eds.), CLS 39-2: The Panels. Papers from the 39th Annual Meeting of the Chicago Linguistic Society, Chicago, University of Chicago Press, 2007, págs. 238-258.

De Cesare, A. M., "Gli avverbi paradigmatizzanti”, en A. Ferrari et al., L'interfaccia lingua-testo. Natura e funzioni dell' articolazione informativa dell'enunciato, Alessandria, Edizioni dell'Orso, 2008, págs. 340-361.

De Cesare, A. M., "La posizione del soggetto tra scritto e parlato: un'analisi alla luce dei casi 'anche + soggetto", en A. Ferrari (ed.), Sintassi storica e sincronica, Subordinazione, coordinazione, giustapposizione. Atti del X Congresso della Società Internazionale di Linguistica e Filologia Italiana (Basilea, 30.6-3.7.2008), Firenze, Cesati, 2009, págs. 955-970.

De Cesare, A. M., "On the focusing function of focusing adverbs. A discussion based on Italian data", en E. Hentschel (ed.), 40 Jahre Partikelforschung/40 Years Particle Research, Linguistik online 44/4 (2010) <www.linguistik-online.com>.

De Cesare, A. M. - Borreguero, M., "The contribution of the Basel Model to the description of the polifunctionality of discourse markers: It. anche, Fr. aussi, Sp. también", en S. Pons Bordería (ed.), Models of discourse segmentation. Explorarions across Romance Languages, Amsterdam, John Benjamins, 2014, págs. 55-94.

Dimroth, Сн., Klein, W., "Fokuspartikeln in Lernervarietäten. Ein Analyserahmen und einige Beispiele", Zeitschrift für Literaturwissenschaft und Linguistik, 104 (1996), págs. 73-114.

FERRARI, A., Le ragioni del testo. Aspetti sintattici interpuntivi dell italiano contemporaneo, Firenze, Accademia della Crusca, 2003.

Ferrari, A., "Connettivi e struttura del testo. Oltre la semantica lessicale", en I. Korzen (ed.), Lingua, cultura e intercultura: l'italiano e le altre lingue (=Copenhagen Studies in Language, 31), Copenhagen, Samfundslitteratur Press, 2005, págs. 191-204.

Ferrari, A., "Congiunzioni frasali, congiunzioni testuali e preposizioni: stessa logica, diversa testualità", en E. Cresti (ed.), Prospettive nello studio del lessico italiano, Atti del 
IX Congresso della Società Internazionale di Linguistica e Filologia Italiana, (Firenze, 14-17.6.2006), Firenze, Firenze University Press, 2008, págs. 411-417.

Ferrari, A., "Connettivi e gerarchie testuali tra morfologia, sintassi e punteggiatura. Note a partire dall'italiano", en M. Borreguero - S. Gómez-Jordana (eds.), Marqueurs $d u$ discours dans les langues romanes. Limoges, Lambert-Lucas, 2015, págs. 129-148.

FERrari, A. et al, L'interfaccia lingua-testo. Natura e funzioni dell'articolazione informativa dell'enuciato, Alessandria, Edizioni dell'Orso, 2008.

Ferrari, A. - Borreguero Zuloaga, M., La interfaz lengua-texto. Un modelo de estructura informativa, Madrid, Biblioteca Nueva, 2015.

Ferrari, A. - De Cesare, A. M. 'Linterprétation de l'adverbe italien proprio entre lexique, syntaxe et textualité", en A. Auchlin et al. (eds.), Structures et Discours. Mélanges offerts à Eddy Roulet, Québec, Éditions Nota Bene, 2004, págs. 195-210.

Ferrari, A. - Mandelli, M. "Note sull'impiego dei connettivi nei notiziari accademici del corpus Athenaeum", en M. Barbera et al. (eds.), Corpora e linguistica in rete, Perugia, Guerra, 2007, págs. 183-198.

Ferrari, A. - Ricci, C., "Valeur d'emploi, portée, relief. Observations descriptives et explicatives sur la relation entre position et inteprétation des connecteurs ", en $\mathrm{S}$. Hancil (ed.), Le role de l'Affect dans les marqueurs discursifs, Rennes, Publications de 1'Université de Rouen et du Havre (PURH), 2011, págs. 41-62.

Ferrari, A. - Rossari, C., "De donc à dunque e quindi: les connexions par raisonnement inférentiel”, Cabiers de Linguistique Française, 15 (1994), págs. 7-49.

Ferrari, A. (ed.), La lingua nel testo, il testo nella lingua, Torino, Istituto dell'Atlante Linguistico Italiano, 2004.

Ferrari, A. (ed.), Rilievi. Le gerarchie semantico-pragmatiche di alcuni tipi di testo, Firenze, Cesati, 2005.

Ferrari, A.- Giammatteo, M. - Albano, H., "Operadores de foco: el caso de incluso, basta, solo y aun", Cuadernos de la ALFAL, 3 (2011), págs. 30-41.

Flamenco García, L., "Las construcciones concesivas y adversativas”, en I. Bosque V. Demonte (coords.), Gramática descriptiva de la lengua española, Madrid, Espasa, 1999, págs. 3805-3878.

Fuentes Rodríguez, C., "Pragmática y relación intratextual: el caso de basta, incluso y ni siquiera”, ELUA 4 (1987), págs. 159-176.

Fuentes Rodríguez, C., Diccionario de conectores y operadores del español, Madrid, Arco Libros, 2009.

García Negroni, M. M., "Cuando la excepción refuerza la argumentación. A propósito del uso exceptivo de même y de incluso", Téleme, Revista Complutense de Estudios Franceses 13 (1998), págs. 207-218.

Garrido Medina, J., “Operadores epistémicos y conectores contextuales”, en H. Haverkate et al. (eds.), Diálogos hispánicos 12. Aproximaciones pragmalingüísticas al español, Amsterdam, Rodopi, 1993, págs. 5-50.

Gast, V. - van der Auwera, J., "Scalar Additive Operators in the Languages of Europe”, Language 87:1 (2011), págs. 2-54. 
GuIL, P., "La interfaz lengua-texto: observaciones contrastivas", comunicación presentada al Convegno internazionali di studi Linguistica testuale. Teorie, metodi, fenomeni, strutture, Basilea, 2-4 julio de 2014.

KaY, P., "Even”, Linguistics and Philosophy, 13 (1990), págs. 59-111.

KöNIG, E., The meaning of focus particles: a comparative perspective, London-New York, Routledge, 1991.

KöNIG, E., "Focus particles", en J. Jacbos - A. von Stechow, - W. Sternfeld - Th. Venemman (eds.), Syntax. Ein internationales Handbuch zeitgenössiger Forschung. An interntional handbook of contemporary research, Berlin / New York, W. de Gruyter, 1993, págs 978-987.

Kovacci, O., "El adverbio", en I. Bosque - V.Demonte (coord.), Gramática descriptiva de la lengua española, Madrid, Espasa-Calpe, 1991, vol. I, págs. 705-786.

Hopper, P. - Traugott, E. C., Gramaticalization, Cambridge, Cambridge University Pres, 1993 [2003], 2nd ed.

La Forgia, L., "Alcune osservazioni sui focalizzatori”, Studi italiani di linguistica teorica ed applicata XXXV:3 (2006), págs. 359-383.

Lambrecht, K., Information structure and sentence form. Topic, focus, and the mental representations of discourse referents, Cambridge, Cambridge University Press, 1994.

Lenarduzzi, R., "El operador anche del italiano y sus formas equivalentes en español", Annali di Ca' Foscari, XXXIV:1-2 (1995), págs. 197-216.

Lenarduzzi, R., "Un estudio contrastivo italiano / español: los adverbios temporales sempre (it.) / siempre (esp.)", en S. Serafin (ed.), Un lume nella notte. Studi di iberistica che gli allievi ed amici dedicano a Giuseppe Bellini. Roma, Bulzoni, 1997a, págs. 143-153.

Lenarduzzi, R., "Un estudio contrastivo italiano / español: los adverbios temporales mai (it.) / nunca (esp.)", Studi di letteratura ispano-americana 30, 1997b, págs. 7-19.

López Serena, A.- Loureda Lamas, Ó., "La reformulación discursiva entre lo oral y lo escrito: una aproximación teórica y experimental”, Oralia, 16 (2013), págs. 221-258.

LOUREDA LAMAs, Ó. et al., "La partícula focal incluso: una aproximación experimental", en U. Wienen - V. Atayan (eds.), Sprache, Rhetorik, Translation: Festschrift für Alberto Gil zu seinem 60. Geburtstag, Frankfurt, Peter Lang, 2013, págs. 55-73.

Mandelli, M., "Coordinazione frasale e coodinazioni testuali: il caso della congiunzione $e$ ", en A. Ferrari (ed.), La lingua nel testo, il testo nella lingua, Torino, Istituto dell'Atlante Linguistico Italiano, 2004, págs. 117-143.

Mandelli, M., "Quella $e$ a inizio di frase...: scarti prosodici e interpuntivi tra elementi coordinati”, en A. Ferrari (ed.), Parole frasi testi, tra scritto e parlato (= Cenobio LV:3), 2006, págs. 231-240.

Mandelli, M., "In effetti nel testo", en E. Cresti (ed.), Prospettive nello studio del lessico italiano. Atti del IX Congresso della Società Internazionale di Linguistica e Filologia Italiana (Firenze, 14-17.6.2006), Firenze, Firenze University Press, 2008, págs. 439-444.

Mederos Martín, H., Procedimientos de cohesión en el español actual, Santa Cruz de Tenerife, Publicaciones Científicas del Excmo. Cabildo Insular de Tenerife. 
Montolío, E., "Las construcciones condicionales”, en I. Bosque - V. Demonte (coords.), Gramática descriptiva de la lengua española, Madrid, Espasa, 1999, vol. III, págs. 3643-3737.

Nøцке, H., Les adverbes paradigmatisants: fonction et analyse (= Revue Romane 23), Copenhagen, Akademisk Forlag, 1983.

Pons Bordería, S. (ed.), Models of discourse segmentation. Explorations across Romance Languages, Amsterdam, John Benjamins, 2014.

Portolés, J., "Escalas informativas aditivas. Pruebas del español”, Spanish in Context 4:2 (2007), págs. 135-157.

Portolés, J., "Incluso”, en A. Briz - S. Pons - J. Portolés (dirs.), Diccionario de partículas del español, <www.dpde.es> [2008].

PortolÉs, J., "Alternativas convocadas por partículas discursivas”, Español actual, 92 (2009), págs. 47-68.

Portolés, J., "Los marcadores del discurso y la estructura informativa”, en Ó. Loureda E. Acín (eds.), Los estudios sobre marcadores del discurso en español, hoy. Madrid, Arco/ Libros, 2010, págs. 281-326.

PortolÉs, J., "Les échelles additives avec además”, en J. C. Anscombre et al. (dirs.), Voix et marqueurs du discours: des connecteurs à l'argument d'autorité, Lyon, ENS Éditions, 2011, págs. 103-118.

PortolÉs, J., “Argumentación y oralidad: el caso de además”, Oralia, 16 (2013), págs. 283-303.

Real Academia Española, Corpus de Referencia del Español Actual (CREA), <www. rae.es>.

Real Academia Española, Asociación de Academias de la Lengua Española, Nueva gramática de la lengua española, Madrid, Espasa, 3 vols., 2009.

Ricca, D., "Osservazioni preliminari sui focalizzatori in italiano”, en N. Dittmar - A. Giacalone Ramat (eds.), Grammatica e discorso. Studi sull'acquisizione dell'italiano $e$ del tedesco. Grammatik und Diskurs. Studien zum Erwerb des Deutschen und des Italienischen, Tübingen, Stauffenburg, 1999, 146-164.

Ricci, C., "I connettivi nel testo", en A. Ferrari et al., L'interfaccia lingua-testo. Natura e funzioni dell'articolazione informativa dell'enunciato, Alessandria, Edizioni dell'Orso, 2008, págs. 362-387.

Roотн, M.E., Association with Focus, Doctoral Thesis, University of Massachusetts at Amherst, 1985. <http//:eCommons.library.cornell.edu>.

Roulet, E. et al., L'articulation du discourse en français contemporain, Bern, Lang, 1985.

Roulet, E. et al., Un modèle et un instrument d'analyse de l'organisation du discourse, Bern, Lang, 2001.

Sabatini, F. - Coletti, V., DISC. Dizionario italiano Sabatini Coletti, Firenze, Giunti, 1997.

Sainz González, M. E., "También/anche: estudio semántico contrastivo", en G. Bazzocchi - M. P. Capanaga Caballero (eds.), Mediación lingüística de lenguas afines: español/italiano. Bologna, Gedit Edizioni, 2006a, págs. 23-45.

Sainz González, M. E., "También / tampoco: marcadores de modalidad deóntica”, Annali di Ca’ Foscari XLV, I:2 (2006b), págs. 267-288. 
Santos Río, L., Diccionario de partículas del español, Salamanca, Luso-Española, 2003.

Schieman, A., "Adverbial indizierte Implikationen: eine argumentationsbasierte Analyse von persino/perfino", en V. Atayan - D. Pierazzini (eds.), Argumentation: théorie - langue - discours. Frankfurt, Peter Lang, 2009, págs. -222.

Schwenter, S., "Lo relativo y lo absoluto de las partículas escalares incluso y hasta", Oralia, 3 (2000), págs. 169-198.

TAGLicht, J.,"Focus and background“, en J.Jacobs et al. (eds.), Syntax. Ein internationales Handbuch zeitgenössischer Forschung / An International Handbook of Contemporary Research, Berlin/New York, W. de Gruyter, 1993, págs. 998-1006.

TAм, L., Dizionario spagnolo-italiano. Diccionario italiano-español, Milano, Hoepli, 1997.

Traugott, E., "The semantic development of scalar focus modifiers", en A. van Kemenade - B. Los (eds.), Handbook of the History of English. London, Blackwell, 2006.

Visconti, J., "Sintassi e uso delle particelle perfino, persino e addirittura in italiano antico", en M. Dardano - G. Frenguelli (eds.), SintAnt. La sintassi dell'italiano antico. Atti del Convegno internazionale di studi (Università "Roma Tre", 18-21 settembre 2002), Roma, Aracne, 2004, págs. 445-463.

VIsconTI, J., "On the origins of scalar particles in Italian", Journal of Historical Pragmatics 6: 2 (2005), págs. 237-261.

Wilkinson, K., "The scope of even", Natural language semantics 4 (1996), págs. 193-215. Zampese, L., "La struttura informativa degli articoli di cronaca: natura e funzioni dell'Unità di Quadro", en A. Ferrari (ed.), Rilievi. Le gerarchie semantico-pragmatiche di alcuni tipi di testo, Firenze, Cesati, 2006, págs. 173-216.

\section{CORPUS}

CREA: Real Academia Española, Corpus de referencia del español actual. <www.rae.es> CORIS: Università di Bologna, Corpus di Italiano Scritto. <corpora.dslo.unibo.it> 
